\title{
Quantum transport and utilization of free energy in protein $\alpha$-helices
}

\author{
Danko D. Georgiev ${ }^{1}$ and James F. Glazebrook ${ }^{2}$ \\ ${ }^{1}$ Institute for Advanced Study, 30 Vasilaki Papadopulu Str., Varna 9010, Bulgaria. E-mail: \\ danko.georgiev@mail.bg \\ ${ }^{2}$ Department of Mathematics and Computer Science, Eastern Illinois University, \\ Charleston, Illinois 61920-3099, USA. E-mail: jfglazebrook@eiu.edu
}

\begin{abstract}
The essential biological processes that sustain life are catalyzed by protein nanoengines, which maintain living systems in far-from-equilibrium ordered states. To investigate energetic processes in proteins, we have analyzed the system of generalized Davydov equations that govern the quantum dynamics of multiple amide I exciton quanta propagating along the hydrogen-bonded peptide groups in $\alpha$-helices. Computational simulations have confirmed the generation of moving Davydov solitons by applied pulses of amide I energy for protein $\alpha$-helices of varying length. The stability and mobility of these solitons depended on the uniformity of dipole-dipole coupling between amide I oscillators, and the isotropy of the exciton-phonon interaction. Davydov solitons were also able to quantum tunnel through massive barriers, or to quantum interfere at collision sites. The results presented here support a non-trivial role of quantum effects in biological systems that lies beyond the mechanistic support of covalent bonds as binding agents of macromolecular structures. Quantum tunneling and interference of Davydov solitons provide catalytically active macromolecular protein complexes with a physical mechanism allowing highly efficient transport, delivery, and utilization of free energy, besides the evolutionary mandate of biological order that supports the existence of such genuine quantum phenomena, and may indeed demarcate the quantum boundaries of life.
\end{abstract}

Keywords: biological order; Davydov soliton; protein $\alpha$-helix; quantum interference; quantum tunneling

\section{Introduction}

Living organisms are considered as open physical systems which utilize the availability of free energy to maintain homeostasis, respond to stimuli, adapt to their environment, grow, reproduce, and to evolve [1-3]. All of these biological functions are implemented by the largescale participation and interaction of proteins. The high versatility of protein functions is achieved by linear polymerization of 20 different standard amino acids into polypeptide chains [4] (noteworthy, non-standard amino acids also exist, cf. [5]). The linear sequence of amino acids in polypeptides is often referred to as the primary structure. The primary structure folds into two main types of hydrogen-bonded secondary structures, $\alpha$-helices and $\beta$-sheets 6,7$]$. Other, special helical secondary structures, such as the $3_{10}$-helix and the $\pi$-helix, are also found in proteins (for review of their functional importance see [8,9]). The ensuing organization of 
secondary structures through hydrogen bonding, ionic bonding, dipole-dipole interactions, London dispersion forces, or through covalent disulfide bonds, provides each protein with its own tertiary structure that is uniquely shaped. Further quaternary assembly of multiple protein subunits provides the means for evolutionary design of nano-engines equipped with multiple active sites, including an adenosine triphosphate (ATP) hydrolytic site for release of free energy, an active catalytic site for converting the released energy into biologically useful work, and a number of allosteric sites for regulation of protein activity. This permits utilizing biochemical energy stored in high-energy ATP pyrophosphate bonds to fuel bio-processes such as protein-assisted directed motion, synthesis of biomolecules, or for transporting biochemical substances across lipid membranes.

The primary importance of proteins for life is reflected in their name: the term "protein" comes from the Greek words "protos", meaning "first", or "proteos", meaning "first of all". In addition to being major catalysts in biological processes, proteins may indeed have been the constituents of the very first physical systems of life. While in modern organisms genetic information for protein production is stored in nucleic acids, recent stochastic simulations support the protein foldamer hypothesis for abiotic origin of life [10]. In particular, short protein sequences composed of hydrophobic and polar amino acids were found to collapse into relatively compact structures with exposed hydrophobic surfaces, which in turn catalyze the elongation of other such hydrophobic/polar protein polymers [10]. Once the first living replicators were stochastically assembled, natural selection would have kicked in and selected those replicators that exploit available energy sources in the most efficient way for the purposes of reproduction. Eventually, after 3.5 billion years of evolution [11], modern day organisms are capable of the effective use of energy as released by single ATP molecules so as to execute highly specialized biological processes, including single steps of the kinesin motor on cytoskeletal protein railways [12,13, or phosphorylation of single amino acid residues in voltage-gated ion channels for modulation of the channel electric conductance [14, 15].

In living systems, energy is transferred in minute quantities because higher energy densities are detrimental to the delicate and fragile structures [16. While the behavior of all molecules is fundamentally described by quantum mechanics, the highly efficient utilization of energy by proteins suggests that quantum effects may play a non-trivial role, and one that lies beyond the deterministic, mechanistic structural support of covalent bonds that bind macromolecules together 17-24]. In this present paper we undertake studying the quantum dynamics resulting from the generalized Davydov Hamiltonian for the transport of energy by multiple amide I quanta in protein $\alpha$-helices. We show that the resulting system of differential equations admits soliton solutions, for which the corresponding waveforms preserve their shapes during propagation, reflect from protein ends, tunnel through massive barriers, and interfere quantum mechanically at collision points to produce sharply focused peaks of concentrated energy. These instances of quantum phenomena appear to be instrumental in delivering biochemical energy to active catalytic sites, and moreover, are expected to be indispensable for the theoretical basis supporting the quantum boundaries of life. Indeed, in making this contribution to the currently burgeoning science of quantum biology, we are indebted to Schrödinger's astonishing insight which predicted quantum mechanical effects as ubiquitous within living systems [25. Presently there is evidence of these effects occurring in several cases: photosynthesis (coherence), avian navigation (entanglement), olfaction (tunneling), the kinetic isotope effect in enzymatic reactions (tunneling), as particular instances (reviewed in 26, 27]; see also earlier works such as [28 31]). 


\section{Protein $\alpha$-helix structure and infrared spectra}

Protein polypeptide chains are linear polymers that are assembled from a repertoire of 20 different standard amino acids joined together through peptide bonds from $\mathrm{N}$-terminus to $\mathrm{C}$ terminus. The identity of each amino acid is determined by its side chain, known as an $\mathrm{R}$ group. The chemical structure of a generic tripeptide is shown below.

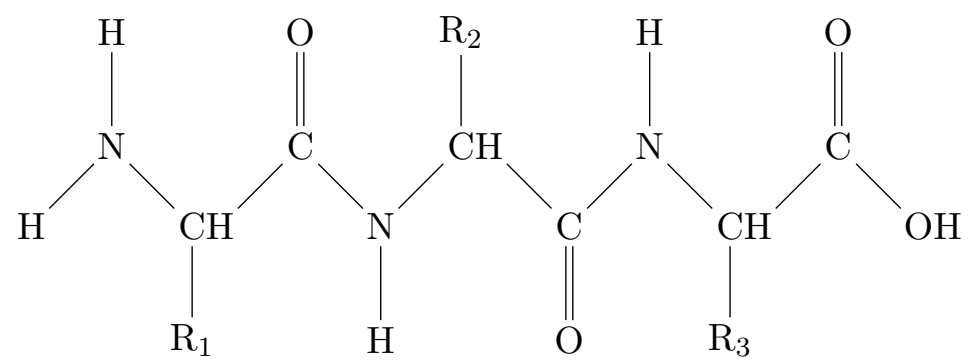

For the construction of protein molecular machines, the flexibility of their polypeptide chains is essential [32]. This allows for organizing the polypeptide primary structure into ordered secondary structural elements such as the protein $\alpha$-helix. Linus Pauling's prediction of the protein $\alpha$-helix in 1951 is one of the greatest achievements in structural biology [33]. For his accomplishments in revealing the three-dimensional geometry of protein secondary structural elements, Pauling was awarded the 1954 Nobel Prize in Chemistry. Besides revealing the protein mysteries of life, it is perhaps not surprising that this distinguished fellow was also actively involved in the preservation of life itself, which subsequently earned him the Nobel Peace Prize in 1962, thus making Pauling the only person to have been awarded two unshared Nobel Prizes [34].

Protein $\alpha$-helices are right-handed spirals with 3.6 amino acids per turn, in which the $\mathrm{N}-\mathrm{H}$ group of an amino acid is hydrogen-bonded with the $\mathrm{C}=\mathrm{O}$ group of the amino acid that appears four residues earlier in the polypeptide chain (Fig. 1). In fact, for $\alpha$-helices there is reliable evidence for the effect of hydrogen bonding on vibrational frequency [35]. The helical structure is supported by three chains of hydrogen-bonded peptide groups $\cdots \mathrm{H}-\mathrm{N}-\mathrm{C}=\mathrm{O} \cdots \mathrm{H}-\mathrm{N}-\mathrm{C}=\mathrm{O} \cdots$ referred to as $\alpha$-helix spines. The typical bond lengths in the peptide group are as follows: $\mathrm{N}-\mathrm{H}$ bond length is $101 \mathrm{pm}, \mathrm{C}-\mathrm{N}$ bond length is $132 \mathrm{pm}$, and $\mathrm{C}=\mathrm{O}$ bond length is $123 \mathrm{pm}[6]$. Because of the resonance between $\mathrm{C}=\mathrm{O}$ and $\mathrm{C}-\mathrm{N}$ bonds, their bond lengths are intermediate between a single and a double bond of the corresponding atoms, and the peptide bonds acquire a planar geometry. Full quantum simulations of electron molecular orbitals in the protein $\alpha$-helix using Kohn-Sham density functional theory [36] are within the capabilities of modern quantum chemistry software applications, but this task would require thousands of hours of running time on a supercomputer [37,38]. Here, we will mainly rely on experimental data obtained from X-ray crystallography, or from the infrared spectroscopy of proteins.

Generally, hydrogen bonding is regarded as significantly instrumental for stabilizing the secondary structure of proteins in two ways: i) through lowering the frequency of stretching vibrations by reducing restoration forces, and ii) by increasing the frequency of bending through increased restoration [35,39]). Under these circumstances, hydrogen bonding can impact the amide functional class by stabilizing its $\left[{ }^{-} \mathrm{O}-\mathrm{C}=\mathrm{N}-\mathrm{H}^{+}\right]$structure over its $[\mathrm{O}=\mathrm{C}-\mathrm{N}-\mathrm{H}]$ structure. Significantly, such bonding reliance can be influenced by the form of amide stretch vibrations. Amide I vibrations mainly arise from the $\mathrm{C}=\mathrm{O}$ stretching vibrations, while supplemented by minor effects elsewhere [35]. As an example, Myshakina et al. [40] demonstrated that the frequency shifts of amide I and amide III bands (see below) may function as significant regulators for hydrogen bonding at the $\mathrm{C}=\mathrm{O}$ and $\mathrm{N}-\mathrm{H}$ sites of certain peptide bonds. Clearly, it is most significant that the proton in such hydrogen bonded systems is a quantum 
entity. Note that quantum nuclear effects may weaken relatively weak hydrogen bonds, while in contrast they may actually fortify the relatively strong ones [41. Relevant in this case is the induced fit method for quantum H-bonding, which supports the molecular interactions for inducing conformational transitions in the binding sites of classes of enzymes [42]. In such a molecular recognition event, the dynamics of tunneling of electrons of proton-acceptor atoms or protons of hydrogen atoms is also significantly instrumental (see below). The tunneling of electrons of proton-acceptor atoms or protons of the hydrogen atoms generating quantum correlations have been studied in Refs. [42,43. Further, in the thermal states of hydrogen bonds, substantial tunneling assisted quantum entanglement can be detected. In particular, if covalent bonding accompanies ionic associates, as is the case for a covalent bond created between electronegative and hydrogen atoms, then quantum entanglement may be subsequently hypothesized for various instances of ligand binding [43].

Fourier-transform infrared (FTIR) spectroscopy allows for experimental measurement, and for plotting the absorption of infrared light by sample material versus the wavelength of the absorbed light. The application of FTIR spectroscopy to proteins has revealed several absorption bands that correspond to polypeptide backbone vibrations [35, 44, 46].

Amide A. Among all absorption bands, most energetic is the amide A band near $3300 \mathrm{~cm}^{-1}$ $(0.41 \mathrm{eV})$, which is due to $>95 \% \mathrm{~N}-\mathrm{H}$ stretch. The amount of this energy correspond exactly to the free energy released from a single ATP molecule. As a result of its exclusive localization on the $\mathrm{N}-\mathrm{H}$ group, however, the amide A band in proteins appears to be insensitive to the secondary structure of the polypeptide backbone [35].

Amide I. Particularly sensitive to the protein secondary structure is the amide I band near $1650 \mathrm{~cm}^{-1}(0.2 \mathrm{eV})$, which is due to $70-85 \% \mathrm{C}=\mathrm{O}$ stretch and $10-20 \% \mathrm{C}-\mathrm{N}$ stretch [44]. The free energy released from a single ATP molecule is sufficient to excite two amide I quanta. Importantly, resonance interaction can occur between two $\mathrm{C}=\mathrm{O}$ oscillators when one of them is in an excited state. For distances over $300 \mathrm{pm}$, the main contribution to the interaction energy is due to transition dipole coupling [44]. The fundamental mechanism that renders the amide I vibration sensitive to secondary structure is the transition dipole coupling, because the coupling between the oscillating dipoles of neighboring amide groups depends upon their relative orientation and their distance 35 . The energy absorbed by a given $\mathrm{C}=\mathrm{O}$ oscillator is readily transferred to nearby oscillators, which leads to delocalized excited states [45].

Amide II. Also sensitive to the secondary structure of proteins, albeit in a less straightforward way, is the amide II band near $1550 \mathrm{~cm}^{-1}(0.19 \mathrm{eV})$, which is due to $40-60 \% \mathrm{~N}-\mathrm{H}$ bend, $18-40 \% \mathrm{C}-\mathrm{N}$ stretch, and $10 \% \mathrm{C}-\mathrm{C}$ stretch.

Amide III. With lowest energy is the amide III band near $1300 \mathrm{~cm}^{-1}(0.16 \mathrm{eV})$, which is due to in-phase combination of $40 \% \mathrm{C}-\mathrm{N}$ stretch and $30 \% \mathrm{~N}-\mathrm{H}$ bend.

As an experimental technology, FTIR spectroscopy can be applied to monitor protein structure in the liquid and dried (lyophilized) state. However, water can interfere with FTIR measurements of protein samples because it is strongly absorbent in the amide I region (how water is distinguished and interpreted in protein chemistry is surveyed in [47, 48]). Consequently, FTIR spectroscopy is best suited for lyophilized (freeze-dried) protein samples. Measurements can also be obtained for protein samples in solution, but a high $(>3 \mathrm{mg} / \mathrm{ml})$ protein concentration is required [49]. We also mention that femtosecond infrared pump-probe spectroscopy has proven to be highly effective for analyzing the amide I band in relationship to the $\mathrm{N}-\mathrm{H}$ vibrations $50-53]$. 


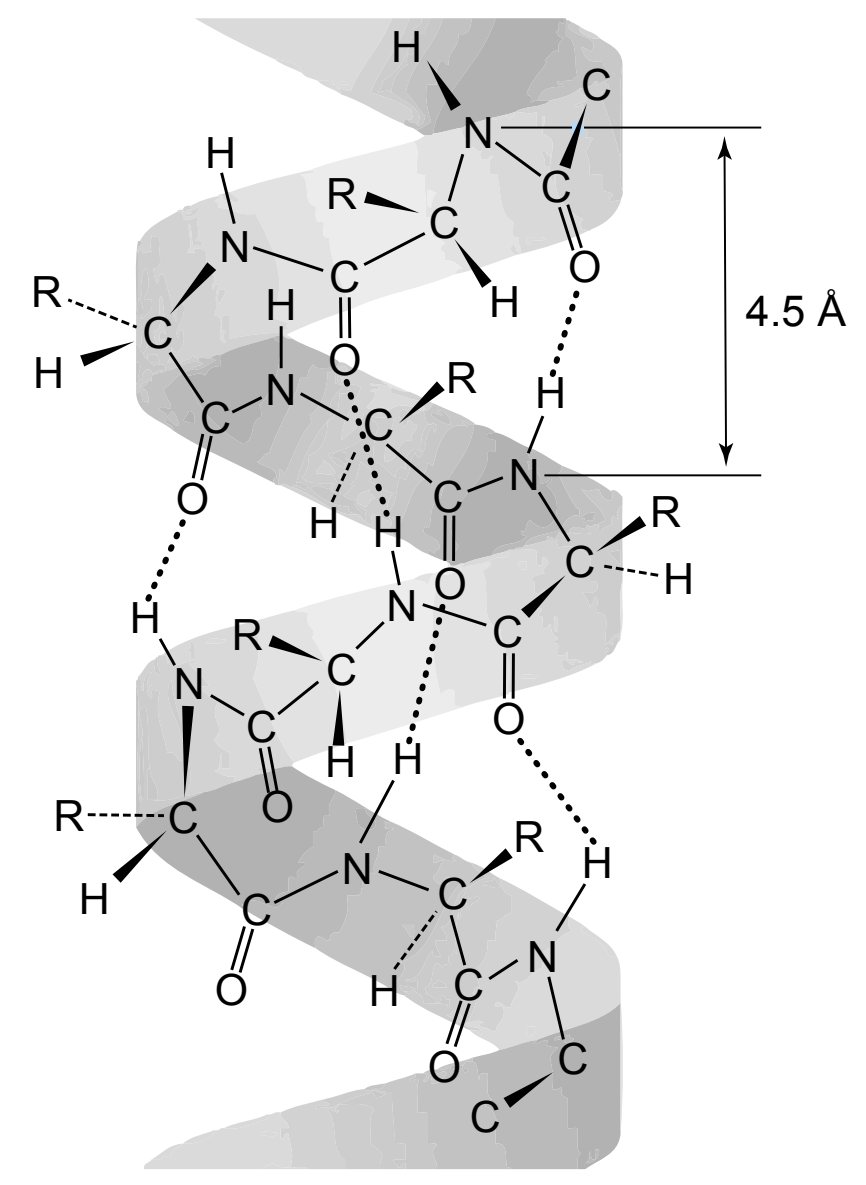

Figure 1: A protein $\alpha$-helix fragment with 3.6 amino acids per turn. The helical structure is supported by three chains of hydrogen-bonded peptide groups $\cdots \mathrm{H}-\mathrm{N}-\mathrm{C}=\mathrm{O} \cdots \mathrm{H}-\mathrm{N}-\mathrm{C}=\mathrm{O} \cdots$ referred to as $\alpha$-helix spines. Modified from Ref. [54].

\section{The generalized Davydov model of protein $\alpha$-helices}

Based on the strong dependence of amide I energy on the protein secondary structure, Alexander Davydov developed a quantum model for the transport of energy in terms of quasiparticles, here referred to as 'solitons' [55-61], where the full atomic complexity of the protein $\alpha$-helix was reduced to the nonlinear interaction between amide I vibrations (excitons), and deformations (phonons) of the lattice of hydrogen bonds that stabilize the helical structure [62 81].

In this work, we model only a single hydrogen-bonded spine in the protein $\alpha$-helix, rather than the 3 -spine structure of the entire helix. In realistic protein $\alpha$-helices, the quantum dynamics of amide I energy in the 3-spine structure will also depend on inter-spine interactions and may give rise to complicated multihump solitons [82,83], which the current study does not consider. For a single $\alpha$-helix spine of hydrogen-bonded peptide groups, the generalized Davydov Hamiltonian is a sum of three parts

$$
\hat{H}=\hat{H}_{\mathrm{ex}}+\hat{H}_{\mathrm{ph}}+\hat{H}_{\mathrm{int}}
$$

respectively for amide I excitons $\hat{H}_{\mathrm{ex}}$, hydrogen-bonded lattice phonons $\hat{H}_{\mathrm{ph}}$, and excitonphonon interaction $\hat{H}_{\text {int }}$. The three parts of the Hamiltonian include only nearest neighbor interactions, and they are formally similar to those featuring in Holstein polaron theory 70 , 


$$
\begin{aligned}
& \hat{H}_{\mathrm{ex}}=\sum_{n}\left[E_{0} \hat{a}_{n}^{\dagger} \hat{a}_{n}-J_{n+1} \hat{a}_{n}^{\dagger} \hat{a}_{n+1}-J_{n} \hat{a}_{n}^{\dagger} \hat{a}_{n-1}\right] \\
& \hat{H}_{\mathrm{ph}}=\frac{1}{2} \sum_{n}\left[\frac{\hat{p}_{n}^{2}}{M_{n}}+w\left(\hat{u}_{n+1}-\hat{u}_{n}\right)^{2}\right] \\
& \hat{H}_{\mathrm{int}}=\chi_{r} \sum_{n}\left(\hat{u}_{n+1}+(\xi-1) \hat{u}_{n}-\xi \hat{u}_{n-1}\right) \hat{a}_{n}^{\dagger} \hat{a}_{n}
\end{aligned}
$$

where the index $n$ counts the peptide groups along the $\alpha$-helix spine, $\hat{a}_{n}^{\dagger}$ and $\hat{a}_{n}$ are the boson creation and annihilation operators for the amide I excitons, $E_{0}$ is the amide I exciton energy, $J_{n}$ is the dipole-dipole coupling energy between the $n$th and $(n-1)$ th amide I oscillator along the spine, $\hat{p}_{n}$ is the momentum operator, $\hat{u}_{n}$ is the displacement operator from the equilibrium position of the peptide group $n, M_{n}$ is the mass of the peptide group $n, w$ is the spring constant of the hydrogen bonds in the lattice [56, 57, 78, $\chi_{r}$ and $\chi_{l}$ are anharmonic parameters arising from the coupling between the amide I exciton and the phonon lattice displacements, respectively to the right or to the left, $\bar{\chi}=\frac{\chi_{r}+\chi_{l}}{2}$, and $\xi=\frac{\chi_{l}}{\chi_{r}}$ is the anisotropy parameter of the exciton-phonon interaction (by construction $\chi_{r} \neq 0$, and $0 \leq \chi_{l} \leq \chi_{r}$ so that $\xi$ varies in the interval $[0,1])[54,80,86$.

The quantum equations of motion for multi-quanta states of amide I energy can be derived from the Hamiltonian (1) with the use of the following generalized ansatz state vector [74:

$$
|\Psi(t)\rangle=|a(t)\rangle|b(t)\rangle=\frac{1}{\sqrt{Q !}}\left[\sum_{n} a_{n}(t) \hat{a}_{n}^{\dagger}\right]^{Q}\left|0_{\mathrm{ex}}\right\rangle e^{-\frac{\imath}{\hbar} \sum_{j}\left(b_{j}(t) \hat{p}_{j}-c_{j}(t) \hat{u}_{j}\right)}\left|0_{\mathrm{ph}}\right\rangle
$$

where

$$
\begin{aligned}
|a(t)\rangle & =\frac{1}{\sqrt{Q !}}\left[\sum_{n} a_{n}(t) \hat{a}_{n}^{\dagger}\right]^{Q}\left|0_{\mathrm{ex}}\right\rangle \\
|b(t)\rangle & =e^{-\frac{\imath}{\hbar} \sum_{j}\left(b_{j}(t) \hat{p}_{j}-c_{j}(t) \hat{u}_{j}\right)}\left|0_{\mathrm{ph}}\right\rangle
\end{aligned}
$$

For ease of notation, the time dependence of $a_{n}(t), b_{n}(t), c_{n}(t),|\Psi(t)\rangle,|a(t)\rangle$ and $|b(t)\rangle$, will henceforth be implicitly understood. Taking the inner product of this generalized ansatz with itself gives

$$
\langle\Psi \mid \Psi\rangle=\left[\sum_{n}\left|a_{n}\right|^{2}\right]^{Q}
$$

which implies

$$
\sum_{n}\left|a_{n}\right|^{2}=1
$$

Verification of (8) requires the multinomial theorem 87

$$
\left[\sum_{n} a_{n}\right]^{Q}=\sum_{k_{1}+k_{2}+\ldots+k_{n}=Q}\left(\begin{array}{c}
Q \\
k_{1}, k_{2}, \ldots, k_{n}
\end{array}\right) a_{1}^{k_{1}} a_{2}^{k_{2}} \ldots a_{n}^{k_{n}}
$$

where the multinomial coefficient is

$$
\left(\begin{array}{c}
Q \\
k_{1}, k_{2}, \ldots, k_{n}
\end{array}\right)=\frac{Q !}{k_{1} ! k_{2} ! \ldots k_{n} !}
$$


with $k_{1}+k_{2}+\ldots+k_{n}=Q$. Then the inner product of the ansatz is

$$
\langle\Psi \mid \Psi\rangle=\frac{1}{Q !}\left\langle 0_{\mathrm{ex}}\left|\left[\sum_{n^{\prime}} a_{n^{\prime}}^{*}(t) \hat{a}_{n^{\prime}}\right]^{Q}\left[\sum_{n} a_{n}(t) \hat{a}_{n}^{\dagger}\right]^{Q}\right| 0_{\mathrm{ex}}\right\rangle\langle b \mid b\rangle
$$

Taking into account that a non-zero result is only possible for creation and annihilation operators with same powers at a given site, together with $\langle b \mid b\rangle=1$ and

$$
\left\langle 0_{\mathrm{ex}}\left|\left(a_{n}^{*} \hat{a}_{n}\right)^{k_{n}}\left(a_{n} \hat{a}_{n}^{\dagger}\right)^{k_{n}}\right| 0_{\mathrm{ex}}\right\rangle=\left(\left|a_{n}\right|^{2}\right)^{k_{n}} k_{n} !
$$

we obtain

$$
\begin{aligned}
\langle\Psi \mid \Psi\rangle=\frac{1}{Q !} \sum_{k_{1}+k_{2}+\ldots+k_{n}=Q}\left(\frac{Q !}{k_{1} ! k_{2} ! \ldots k_{n} !}\right)^{2}\left(\left|a_{1}\right|^{2}\right)^{k_{1}}\left(\left|a_{2}\right|^{2}\right)^{k_{2}} \ldots\left(\left|a_{n}\right|^{2}\right)^{k_{n}} \\
\quad \times\left\langle 0_{\mathrm{ex}}\left|\hat{a}_{1}^{k_{1}} \hat{a}_{2}^{k_{2}} \ldots \hat{a}_{n}^{k_{n}}\left(\hat{a}_{1}^{\dagger}\right)^{k_{1}}\left(\hat{a}_{2}^{\dagger}\right)^{k_{2}} \ldots\left(\hat{a}_{n}^{\dagger}\right)^{k_{n}}\right| 0_{\mathrm{ex}}\right\rangle \\
=\sum_{k_{1}+k_{2}+\ldots+k_{n}=Q} \frac{Q !}{k_{1} ! k_{2} ! \ldots k_{n} !}\left(\left|a_{1}\right|^{2}\right)^{k_{1}}\left(\left|a_{2}\right|^{2}\right)^{k_{2}} \ldots\left(\left|a_{n}\right|^{2}\right)^{k_{n}}=\left[\sum_{n}\left|a_{n}\right|^{2}\right]^{Q}
\end{aligned}
$$

For $Q=1$, the generalized ansatz $|\Psi(t)\rangle$ reduces to Davydov's original $\left|D_{2}(t)\right\rangle$ ansatz [58, 81], which was studied extensively in several previous works [54,73, 86].

Given that the ansatz (5) is a suitable approximation to the exact solution of the Schrödinger equation [88], we obtain

$$
\imath \hbar \frac{d}{d t}|\Psi\rangle=\hat{H}|\Psi\rangle
$$

For a given peptide group $n$, the expectation values for the exciton number operator $\hat{N}_{n}=\hat{a}_{n}^{\dagger} \hat{a}_{n}$, phonon displacement operator $\hat{u}_{n}$ and phonon momentum operator $\hat{p}_{n}$ are

$$
\begin{aligned}
\left\langle\hat{N}_{n}\right\rangle & =\left\langle\Psi\left|\hat{N}_{n}\right| \Psi\right\rangle=Q\left|a_{n}\right|^{2} \\
\left\langle\hat{u}_{n}\right\rangle & =\left\langle\Psi\left|\hat{u}_{n}\right| \Psi\right\rangle=b_{n} \\
\left\langle\hat{p}_{n}\right\rangle & =\left\langle\Psi\left|\hat{p}_{n}\right| \Psi\right\rangle=c_{n}
\end{aligned}
$$

The verification of (15) requires the use of

$$
\left\langle 0_{\mathrm{ex}}\left|\left(a_{n}^{*} \hat{a}_{n}\right)^{k_{n}} \hat{a}_{n}^{\dagger} \hat{a}_{n}\left(a_{n} \hat{a}_{n}^{\dagger}\right)^{k_{n}}\right| 0_{\mathrm{ex}}\right\rangle=\left(\left|a_{n}\right|^{2}\right)^{k_{n}} k_{n} k_{n} !
$$

Substitution of the ansatz (5) in $\left\langle\Psi\left|\hat{N}_{n}\right| \Psi\right\rangle$, and using the multinomial theorem (10), gives

$$
\begin{aligned}
\left\langle\Psi\left|\hat{N}_{n}\right| \Psi\right\rangle & =\frac{1}{Q !} \sum_{k_{1}+k_{2}+\ldots+k_{n}=Q}\left(\frac{Q !}{k_{1} ! k_{2} ! \ldots k_{n} !}\right)^{2}\left(\left|a_{1}\right|^{2}\right)^{k_{1}}\left(\left|a_{2}\right|^{2}\right)^{k_{2}} \ldots\left(\left|a_{n}\right|^{2}\right)^{k_{n}} k_{n} k_{1} ! k_{2} ! \ldots k_{n} ! \\
& =\sum_{k_{1}+k_{2}+\ldots+\left(k_{n}-1\right)=Q-1} \frac{Q(Q-1) !}{k_{1} ! k_{2} ! \ldots\left(k_{n}-1\right) !}\left(\left|a_{1}\right|^{2}\right)^{k_{1}}\left(\left|a_{2}\right|^{2}\right)^{k_{2}} \ldots\left(\left|a_{n}\right|^{2}\right)^{\left(k_{n}-1+1\right)} \\
& =Q\left|a_{n}\right|^{2}\left[\sum_{n^{\prime}}\left|a_{n^{\prime}}\right|^{2}\right]^{Q-1}=Q\left|a_{n}\right|^{2}
\end{aligned}
$$


Verification of (16) requires the result that the expectation values of the position and momentum operators of peptide groups with the vacuum are zero, $\left\langle 0_{\mathrm{ph}}\left|\hat{u}_{n}\right| 0_{\mathrm{ph}}\right\rangle=0$ and $\left\langle 0_{\mathrm{ph}}\left|\hat{p}_{n}\right| 0_{\mathrm{ph}}\right\rangle=$ 0, together with the Hadamard lemma:

$$
\begin{aligned}
e^{\hat{A}} \hat{B} e^{-\hat{A}} & =\exp \left(\operatorname{ad}_{\hat{A}}\right)(\hat{B})=\sum_{k=0}^{\infty} \frac{1}{k !}\left(\operatorname{ad}_{\hat{A}}\right)^{k}(\hat{B}) \\
& =\hat{B}+[\hat{A}, \hat{B}]+\frac{1}{2 !}[\hat{A},[\hat{A}, \hat{B}]]+\frac{1}{3 !}[\hat{A},[\hat{A},[\hat{A}, \hat{B}]]]+\ldots
\end{aligned}
$$

where $\operatorname{ad}_{\hat{A}}(\hat{B}) \equiv[\hat{A}, \hat{B}]$ is the adjoint operator. Substitution of the ansatz $[5]$ in $\left\langle\Psi\left|\hat{u}_{n}\right| \Psi\right\rangle$, and application of the Hadamard lemma $(19)$ with the standard quantum commutation relations $\left[\hat{u}_{n}, \hat{p}_{n}\right]=\imath \hbar$ and $\left[\hat{p}_{n}, \hat{u}_{n}\right]=-\imath \hbar$, gives

$$
\begin{aligned}
& \left\langle\Psi\left|\hat{u}_{n}\right| \Psi\right\rangle=\langle a \mid a\rangle\left\langle 0_{\mathrm{ph}}\left|e^{\frac{\imath}{\hbar} \sum_{j}\left(b_{j} \hat{p}_{j}-c_{j} \hat{u}_{j}\right)} \hat{u}_{n} e^{-\frac{\imath}{\hbar} \sum_{j}\left(b_{j} \hat{p}_{j}-c_{j} \hat{u}_{j}\right)}\right| 0_{\mathrm{ph}}\right\rangle \\
& =\left\langle 0_{\mathrm{ph}}\left|e^{\frac{\imath}{\hbar}\left(b_{n} \hat{p}_{n}-c_{n} \hat{u}_{n}\right)} e^{\frac{\imath}{\hbar} \sum_{j \neq n}\left(b_{j} \hat{p}_{j}-c_{j} \hat{u}_{j}\right)} \hat{u}_{n} e^{-\frac{\imath}{\hbar} \sum_{j \neq n}\left(b_{j} \hat{p}_{j}-c_{j} \hat{u}_{j}\right)} e^{-\frac{\imath}{\hbar}\left(b_{n} \hat{p}_{n}-c_{n} \hat{u}_{n}\right)}\right| 0_{\mathrm{ph}}\right\rangle \\
& =\left\langle 0_{\mathrm{ph}}\left|e^{\frac{\imath}{\hbar}\left(b_{n} \hat{p}_{n}-c_{n} \hat{u}_{n}\right)} e^{\frac{\imath}{\hbar} \sum_{j \neq n}\left(b_{j} \hat{p}_{j}-c_{j} \hat{u}_{j}\right)} e^{-\frac{\imath}{\hbar} \sum_{j \neq n}\left(b_{j} \hat{p}_{j}-c_{j} \hat{u}_{j}\right)} \hat{u}_{n} e^{-\frac{\imath}{\hbar}\left(b_{n} \hat{p}_{n}-c_{n} \hat{u}_{n}\right)}\right| 0_{\mathrm{ph}}\right\rangle \\
& =\left\langle 0_{\mathrm{ph}}\left|e^{\frac{\imath}{\hbar}\left(b_{n} \hat{p}_{n}-c_{n} \hat{u}_{n}\right)} \hat{u}_{n} e^{-\frac{\imath}{\hbar}\left(b_{n} \hat{p}_{n}-c_{n} \hat{u}_{n}\right)}\right| 0_{\mathrm{ph}}\right\rangle \\
& =\left\langle 0_{\mathrm{ph}}\left|\hat{u}_{n}+\left[\frac{\imath}{\hbar}\left(b_{n} \hat{p}_{n}-c_{n} \hat{u}_{n}\right), \hat{u}_{n}\right]\right| 0_{\mathrm{ph}}\right\rangle \\
& =\left\langle 0_{\mathrm{ph}}\left|\hat{u}_{n}\right| 0_{\mathrm{ph}}\right\rangle+\left\langle 0_{\mathrm{ph}}\left|b_{n}\right| 0_{\mathrm{ph}}\right\rangle=b_{n}
\end{aligned}
$$

where we used $\langle a \mid a\rangle=1$ as implied from (8) and (9). Verification of (17) is analogous 89 (only the last two steps are shown):

$$
\left\langle\Psi\left|\hat{p}_{n}\right| \Psi\right\rangle=\left\langle 0_{\mathrm{ph}}\left|\hat{p}_{n}+\left[\frac{\imath}{\hbar}\left(b_{n} \hat{p}_{n}-c_{n} \hat{u}_{n}\right), \hat{p}_{n}\right]\right| 0_{\mathrm{ph}}\right\rangle=\left\langle 0_{\mathrm{ph}}\left|\hat{p}_{n}\right| 0_{\mathrm{ph}}\right\rangle+\left\langle 0_{\mathrm{ph}}\left|c_{n}\right| 0_{\mathrm{ph}}\right\rangle=c_{n}
$$

Identifying $b_{n}$ and $c_{n}$ as quantum expectation values is important for a further application of the Schrödinger equation and its complex conjugate in the form of the generalized Ehrenfest theorem [54], which governs the quantum dynamics of the expectation values

$$
\begin{aligned}
\frac{d}{d t} b_{n} & =\frac{1}{\imath \hbar}\left\langle\Psi\left|\left[\hat{u}_{n}, \hat{H}\right]\right| \Psi\right\rangle \\
\frac{d}{d t} c_{n} & =\frac{1}{\imath \hbar}\left\langle\Psi\left|\left[\hat{p}_{n}, \hat{H}\right]\right| \Psi\right\rangle
\end{aligned}
$$

From the Hamiltonian (1), together with $\left[\hat{u}_{n}, \hat{p}_{n}^{2}\right]=2 \imath \hbar \hat{p}_{n}$, and $\left[\hat{p}_{n}, \hat{u}_{n}^{2}\right]=-2 \imath \hbar \hat{u}_{n}$, we obtain the two commutators to be

$$
\begin{aligned}
& {\left[\hat{u}_{n}, \hat{H}\right]=\imath \hbar \frac{\hat{p}_{n}}{M_{n}}} \\
& {\left[\hat{p}_{n}, \hat{H}\right]=\imath \hbar w\left(\hat{u}_{n-1}-2 \hat{u}_{n}+\hat{u}_{n+1}\right)-\imath \hbar \chi_{r}\left(\hat{a}_{n-1}^{\dagger} \hat{a}_{n-1}+(\xi-1) \hat{a}_{n}^{\dagger} \hat{a}_{n}-\xi \hat{a}_{n+1}^{\dagger} \hat{a}_{n+1}\right)}
\end{aligned}
$$

Substitution in (20) and (21), followed by application of (15), (16) and (17), yields one of Davydov's equations

$$
M_{n} \frac{d^{2}}{d t^{2}} b_{n}=w\left(b_{n-1}-2 b_{n}+b_{n+1}\right)-Q \chi_{r}\left(\left|a_{n-1}\right|^{2}+(\xi-1)\left|a_{n}\right|^{2}-\xi\left|a_{n+1}\right|^{2}\right)
$$

The equation for the amide I probability amplitudes $a_{n}$ can be derived by differentiating the $|\Psi\rangle$ ansatz using the product rule

$$
\imath \hbar \frac{d}{d t}|\Psi\rangle=\left(\imath \hbar \frac{d}{d t}|a\rangle\right)|b\rangle+|a\rangle\left(\imath \hbar \frac{d}{d t}|b\rangle\right)
$$


where (cf. [73, 74])

$$
\begin{aligned}
& \imath \hbar \frac{d}{d t}|a\rangle=\imath \hbar \sqrt{Q}\left(\sum_{n^{\prime}} \frac{d a_{n^{\prime}}}{d t} \hat{a}_{n^{\prime}}^{\dagger}\right) \frac{1}{\sqrt{(Q-1) !}}\left(\sum_{n} a_{n} \hat{a}_{n}^{\dagger}\right)^{Q-1}\left|0_{\mathrm{ex}}\right\rangle \\
& \imath \hbar \frac{d}{d t}|b\rangle=\sum_{n}\left[\frac{d b_{n}}{d t} \hat{p}_{n}-\frac{d c_{n}}{d t} \hat{u}_{n}+\frac{1}{2}\left(b_{n} \frac{d c_{n}}{d t}-\frac{d b_{n}}{d t} c_{n}\right)\right]|b\rangle
\end{aligned}
$$

Next, we use the Schrödinger equation (14) and take the inner product with the state $\frac{1}{\sqrt{Q !}}\langle b|\left\langle 0_{\mathrm{ex}}\right|\left(\hat{a}_{n}\right)^{Q}$ as follows:

$$
\frac{1}{\sqrt{Q !}}\langle b|\left\langle 0_{\mathrm{ex}}\left|\left(\hat{a}_{n}\right)^{Q} \imath \hbar \frac{d}{d t}\right| \Psi\right\rangle=\frac{1}{\sqrt{Q !}}\langle b|\left\langle 0_{\mathrm{ex}}\left|\left(\hat{a}_{n}\right)^{Q} \hat{H}\right| \Psi\right\rangle
$$

Again, a non-zero result is only possible if the creation and annihilation operators at a given site have the same power. Since the operators act at most on two different sites, the use of the multinomial theorem reduces to the binomial one (there are at most two sites with non-zero powers). Straightforward application of binomial coefficients $\left(\begin{array}{l}n \\ k\end{array}\right)=\frac{n !}{k !(n-k) !}$ allows the calculation of individual inner products

$$
\begin{aligned}
& \frac{1}{\sqrt{Q !}}\left\langle 0_{\mathrm{ex}}\left|\left(\hat{a}_{n}\right)^{Q} \frac{d}{d t}\right| a\right\rangle=\frac{1}{\sqrt{Q !}} \frac{Q}{\sqrt{Q}} \frac{d a_{n}}{d t} \frac{1}{\sqrt{(Q-1) !}}\left(\begin{array}{c}
Q-1 \\
Q-1
\end{array}\right) a_{n}^{Q-1}\left\langle 0_{\mathrm{ex}}\left|\left(\hat{a}_{n}\right)^{Q}\left(\hat{a}_{n}^{\dagger}\right)^{Q}\right| 0_{\mathrm{ex}}\right\rangle \\
& =\frac{d a_{n}}{d t} a_{n}^{Q-1} Q \\
& \frac{1}{\sqrt{Q !}}\left\langle 0_{\mathrm{ex}}\left|\left(\hat{a}_{n}\right)^{Q}\right| a\right\rangle=\frac{1}{\sqrt{Q !}} \frac{1}{\sqrt{Q !}}\left(\begin{array}{c}
Q \\
Q
\end{array}\right) a_{n}^{Q}\left\langle 0_{\mathrm{ex}}\left|\left(\hat{a}_{n}\right)^{Q}\left(\hat{a}_{n}^{\dagger}\right)^{Q}\right| 0_{\mathrm{ex}}\right\rangle \\
& =a_{n}^{Q} \\
& \frac{1}{\sqrt{Q !}}\left\langle 0_{\mathrm{ex}}\left|\left(\hat{a}_{n}\right)^{Q} \hat{a}_{n}^{\dagger} \hat{a}_{n}\right| a\right\rangle=\frac{1}{\sqrt{Q !}} \frac{1}{\sqrt{Q !}}\left(\begin{array}{c}
Q \\
Q
\end{array}\right) a_{n}^{Q}\left\langle 0_{\mathrm{ex}}\left|\left(\hat{a}_{n}\right)^{Q} \hat{a}_{n}^{\dagger} \hat{a}_{n}\left(\hat{a}_{n}^{\dagger}\right)^{Q}\right| 0_{\mathrm{ex}}\right\rangle \\
& =a_{n}^{Q} Q \\
& \frac{1}{\sqrt{Q !}}\left\langle 0_{\mathrm{ex}}\left|\left(\hat{a}_{n}\right)^{Q} \hat{a}_{n}^{\dagger} \hat{a}_{n \pm 1}\right| a\right\rangle=\frac{1}{\sqrt{Q !}} \frac{1}{\sqrt{Q !}}\left(\begin{array}{c}
Q \\
Q-1
\end{array}\right) a_{n \pm 1} a_{n}^{Q-1}\left\langle 0_{\mathrm{ex}}\left|\left(\hat{a}_{n}\right)^{Q}\left(\hat{a}_{n}^{\dagger}\right)^{Q}\right| 0_{\mathrm{ex}}\right\rangle \\
& =a_{n \pm 1} a_{n}^{Q-1} Q
\end{aligned}
$$

Substitution of (25) together with the Hamiltonian (1) into (28), together with the expectation values (16) and (17), after cancellation of common factor $a_{n}^{Q-1} Q$ yields Davydov's second equation

$$
\imath \hbar \frac{d}{d t} a_{n}=\gamma(t) a_{n}-J_{n+1} a_{n+1}-J_{n} a_{n-1}+\chi_{r}\left(b_{n+1}+(\xi-1) b_{n}-\xi b_{n-1}\right) a_{n}
$$

where $\gamma(t)$ is a real-valued global term for all sites $n$ given by

$$
\gamma(t)=E_{0}+\frac{1}{Q}\left[W(t)+\frac{1}{2} \sum_{j}\left(b_{j} \frac{d c_{j}}{d t}-\frac{d b_{j}}{d t} c_{j}\right)\right]
$$


The phonon energy $W(t)$ can be computed with the use of the Hadamard lemma (19) again utilizing the zero expectation values of the position and momentum operators of peptide groups with the vacuum, together with $c_{j}=M_{j} \frac{d b_{j}}{d t}$, and the following commutators

$$
\begin{gathered}
{\left[-\frac{\imath}{\hbar} c_{j} \hat{u}_{j}, \frac{1}{2} \frac{\hat{p}_{j}^{2}}{M_{j}}\right]=\frac{c_{j}}{M_{j}} \hat{p}_{j}} \\
{\left[-\frac{\imath}{\hbar} c_{j} \hat{u}_{j}, \frac{c_{j}}{M_{j}} \hat{p}_{j}\right]=\frac{c_{j}^{2}}{M_{j}}} \\
{\left[\frac{\imath}{\hbar}\left(b_{j} \hat{p}_{j}+b_{j+1} \hat{p}_{j+1}\right), \frac{1}{2} w\left(\hat{u}_{j+1}^{2}-2 \hat{u}_{j} \hat{u}_{j+1}+\hat{u}_{j}^{2}\right)\right]=w\left(b_{j+1} \hat{u}_{j+1}-b_{j} \hat{u}_{j+1}-b_{j+1} \hat{u}_{j}+b_{j} \hat{u}_{j}\right)} \\
{\left[\frac{\imath}{\hbar}\left(b_{j} \hat{p}_{j}+b_{j+1} \hat{p}_{j+1}\right), w\left(b_{j+1} \hat{u}_{j+1}-b_{j} \hat{u}_{j+1}-b_{j+1} \hat{u}_{j}+b_{j} \hat{u}_{j}\right)\right]=w\left(b_{j+1}^{2}-2 b_{j} b_{j+1}+b_{j}^{2}\right)} \\
W(t)=\left\langle b\left|H_{\mathrm{ph}}\right| b\right\rangle=W_{0}+\frac{1}{2} \sum_{j}\left[M_{j}\left(\frac{d b_{j}}{d t}\right)^{2}+w\left(b_{j+1}-b_{j}\right)^{2}\right]
\end{gathered}
$$

with $W_{0}=\left\langle 0_{\mathrm{ph}}\left|\hat{H}_{\mathrm{ph}}\right| 0_{\mathrm{ph}}\right\rangle$ denoting the zero-point phonon energy.

In order to make further headway, we note that introduction of a global phase change on the exciton quantum probability amplitudes, namely $a_{n} \rightarrow \bar{a}_{n} e^{-\frac{\imath}{\hbar} \int \gamma(t) d t}$, will not change the quantum probabilities for finding the exciton at a given site

$$
\left|a_{n}\right|^{2}=e^{+\frac{\imath}{\hbar} \int \gamma(t) d t} \bar{a}_{n}^{*} \bar{a}_{n} e^{-\frac{\imath}{\hbar} \int \gamma(t) d t}
$$

This is important because we will not need to transform the first Davydov equation (24). After differentiation of the left-hand side of 29 )

$$
\imath \hbar \frac{d}{d t}\left(\bar{a}_{n} e^{-\frac{\imath}{\hbar} \int \gamma(t) d t}\right)=\imath \hbar e^{-\frac{\imath}{\hbar} \int \gamma(t) d t} \frac{d \bar{a}_{n}}{d t}+\bar{a}_{n} \gamma(t) e^{-\frac{\imath}{\hbar} \int \gamma(t) d t}
$$

followed by cancellation of common terms and relabeling of $\bar{a}_{n}$ back to $a_{n}$, we obtain the following system of gauge transformed quantum equations of motion

$$
\begin{aligned}
\imath \hbar \frac{d}{d t} a_{n} & =-J_{n+1} a_{n+1}-J_{n} a_{n-1}+\chi_{r}\left[b_{n+1}+(\xi-1) b_{n}-\xi b_{n-1}\right] a_{n} \\
M_{n} \frac{d^{2}}{d t^{2}} b_{n} & =w\left(b_{n-1}-2 b_{n}+b_{n+1}\right)-Q \chi_{r}\left(\left|a_{n-1}\right|^{2}+(\xi-1)\left|a_{n}\right|^{2}-\xi\left|a_{n+1}\right|^{2}\right)
\end{aligned}
$$

\section{A computational study}

\subsection{Model parameters}

The system of generalized Davydov equations (33) and (34) for multiple amide I quanta expands the scope of the original Davydov model (cf. 54, 56, 64, 66, 68, 73, 74, 86, 90, 92]) to the extent that it is now capable of answering a number of questions in regard to the quantum boundaries of life. In particular, anisotropy of the exciton-phonon coupling for different values of the parameter $\xi$, nonuniformity of amino acid masses $M_{n}$, and nonuniformity of amide I dipole-dipole coupling energies $J_{n}$ could be directly incorporated in computer simulations and the resulting effects on quantum dynamics could be theoretically characterized. In order to improve on the comparison with a number of previous studies [54, 69, 75, 77, 86], we here apply the following basic model parameters: spring constant of the hydrogen bonds in the lattice $w=13 \mathrm{~N} / \mathrm{m}$ [93], anharmonic parameter for the excitonphonon coupling $\bar{\chi}=35 \mathrm{pN}$ [54], average mass of an amino acid inside the protein $\alpha$-helix 
$M=1.9 \times 10^{-25} \mathrm{~kg} 69$, average amide I dipole-dipole coupling energy $J=0.155 \mathrm{zJ}$ [94], initially unperturbed lattice of hydrogen bonds, and $Q$ non-overlapping Gaussian pulses of amide I energy each spread over 5 peptide groups with quantum probability amplitudes $a_{n}$ given by $\sqrt{\frac{1}{Q}}\{\sqrt{0.099}, \sqrt{0.24}, \sqrt{0.322}, \sqrt{0.24}, \sqrt{0.099}\}$. For simulations with a double soliton, we used $Q-1$ non-overlapping Gaussian pulses, of which the double soliton had a factor of $\sqrt{\frac{2}{Q}}$ whereas the single solitons had a factor of $\sqrt{\frac{1}{Q}}$. For most of the simulations, we used an $\alpha$-helix spine with length of $n_{\max }=40$ peptide groups, which covers a distance of $18 \mathrm{~nm}$.

\subsection{Solitons in short protein $\alpha$-helices}

Davydov's original analysis aimed to establish the existence of soliton (quasiparticle) solutions via derivation of nonlinear Schrödinger equation (NLSE). To achieve that goal, the excitonphonon interaction was taken to be completely isotropic $\xi=1$, and all $J_{n}$ and $M_{n}$ were uniformly replaced with the corresponding average values $J$ and $M$. Davydov further introduced the dimensionless variable $x=\frac{\tilde{x}}{a}$, where $\tilde{x}$ is the distance and $a$ is the spacing between peptide groups [56]. He then approximated the system of discrete functions with continuous ones using the transformation $f(x \pm 1, t) \approx\left(1 \pm \frac{\partial}{\partial x}+\frac{1}{2} \frac{\partial^{2}}{\partial x^{2}}\right) f(x, t) 57$. Lastly, the resulting system of partial differential equations (PDEs) was manipulated into NLSE, which is known to have analytic soliton solutions [90]. However, because a large number of mathematical assumptions enter at each step of Davydov's derivation, it is not known whether the soliton will persist in short protein $\alpha$-helices in the presence of anisotropy and variability of various parameters.

In order to systematize the effect of the protein $\alpha$-helix length upon these solitons, we have integrated numerically the system of Davydov equations with $Q=1$ for completely isotropic exciton-phonon interaction $\xi=1$ and uniform values $J$ and $M$ for all peptide groups $n$. The quantum dynamics of solitons launched from either the N-end or the C-end of the $\alpha$-helix exhibited left-right mirror symmetry (Fig. 2). The solitons moved with velocity of $334 \mathrm{~m} / \mathrm{s}$, which is slower than 1 peptide group per picosecond (PG/ps), $1 \mathrm{PG} / \mathrm{ps}=450 \mathrm{~m} / \mathrm{s}$. The solitons were capable of multiple reflections from the $\alpha$-helix ends without disintegration. In fact, discreteness effects did not manifest even for ultrashort helices whose length was only $n_{\max }=10$ peptide groups (Figs. 2p,f), even though the initial soliton width was already 5 peptide groups, which is half the length of the helix. This reveals that Davydov's intuition in regard to the approximations, was essentially correct; the continuum approximation is valid for $\xi=1$ regardless of the actual protein $\alpha$-helix length and the soliton width.

Next, we have integrated numerically the system of Davydov equations for completely anisotropic exciton-phonon interaction $\xi=0$, which coincides with the model extensively studied by Scott and collaborators $[69,76,78]$. The speed of launched solitons was lower $218 \mathrm{~m} / \mathrm{s}$ and wobbled in time (Fig. 3). In fact, for ultrashort helices whose length was only $n_{\max }=10$ peptide groups, the soliton reflected 6-7 times before settling in the middle of the helix within a timescale of $100 \mathrm{ps}$ (Figs. 3r,f). Thus, the analytic soliton solution that moves at a constant speed fails to capture correctly the quantum dynamics of the system for $\xi=0$.

In actual protein $\alpha$-helices, the case of complete anisotropy of exciton-phonon interaction, $\xi=\frac{\chi_{l}}{\chi_{r}}=0$, cannot be reached as this will require absolutely no interaction of the amide I oscillator with the hydrogen bond to the left $\left(\chi_{l}=0\right)$. From a theoretical perspective, however, letting $\xi=0$ provides a limiting case that may exhibit qualitatively different quantum behavior. Thus, varying $\xi$ within the interval $[0,1]$ in systematic steps could provide a threshold for qualitative change in quantum dynamics. Interestingly, in the current context, such a threshold value exists and this could be set to $\xi=0.02$. The presence of very slight isotropy of the exciton-phonon interaction $\xi=0.02$ was able to restore the linear motion of the soli- 
a

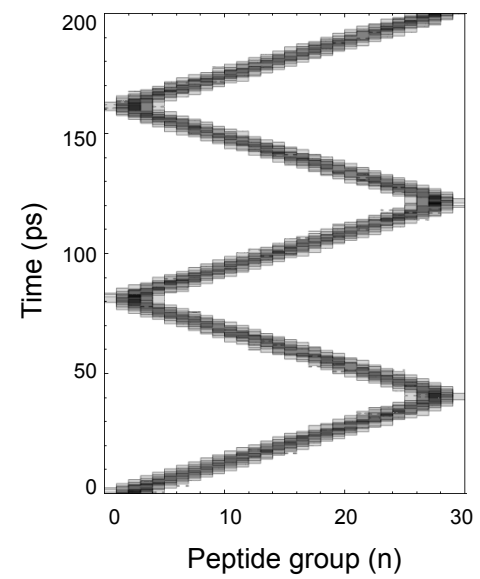

d

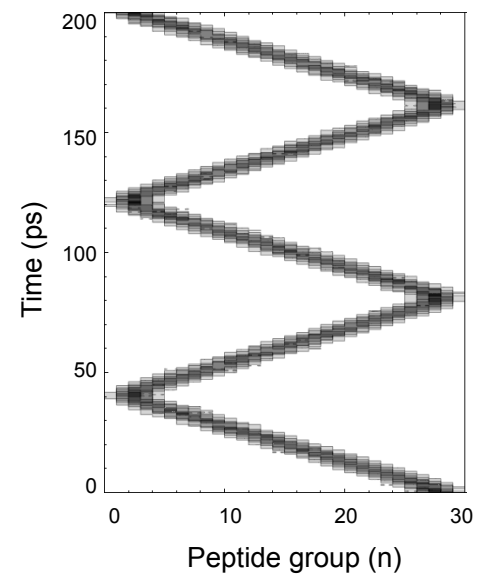

b

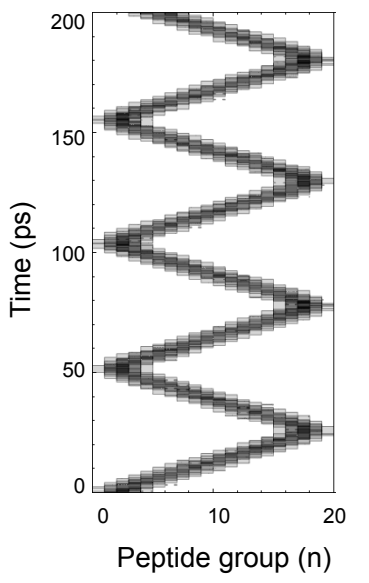

e

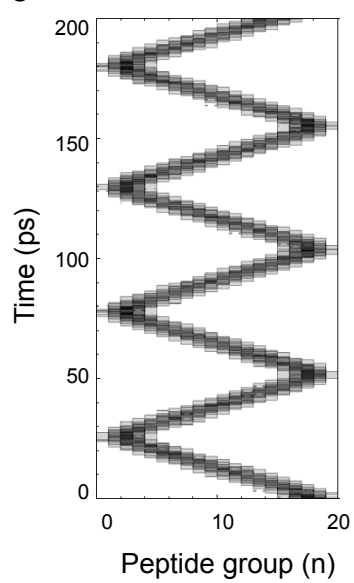

C

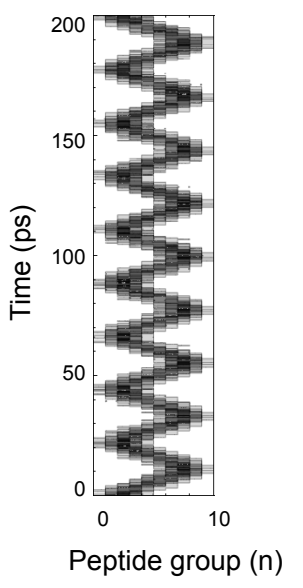

f

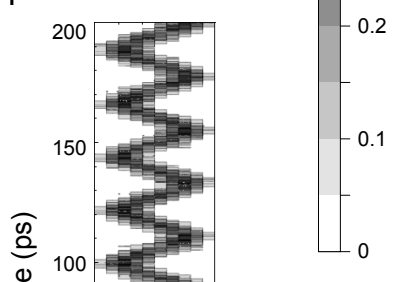

Figure 2: The quantum dynamics of a moving Davydov soliton for completely isotropic exciton-phonon interaction $\xi=1$, with $Q=1$ quantum of amide I energy visualized through the expectation value of the exciton number operator $Q\left|a_{n}\right|^{2}$ at each peptide group $n$. (a-c) Gaussian pulse of amide I energy is applied at the N-end of a short protein $\alpha$-helix spine with varying length, $n_{\max }=30(\mathrm{a}), n_{\max }=20(\mathrm{~b})$ or $n_{\max }=10(\mathrm{c})$. (d-f) Gaussian pulse of amide I energy is applied at the C-end of a short protein $\alpha$-helix spine with varying length, $n_{\max }=30(\mathrm{~d}), n_{\max }=20(\mathrm{e})$ or $n_{\max }=10(\mathrm{f})$.

ton increasing its speed to $241 \mathrm{~m} / \mathrm{s}$ (Fig. 4). The soliton reflected repeatedly in ultrashort helices with $n_{\max }=10$ peptide groups for the whole time period of 200 ps of the simulation (Figs. 4k,f), similarly to the completely isotropic case with $\xi=1$. In other words, for realistic protein $\alpha$-helices the main effect of the anisotropy of exciton-phonon interaction could be to reduce the velocity of propagation.

Collectively, the above results indicate that the Davydov solitons are robust quasiparticles with respect to lattice discreteness, and in turn, with respect to the total length of the protein $\alpha$-helix. Thus, in the process of evolutionary design, and optimization of protein functions in general, it is physically plausible that the lengths of various protein $\alpha$-helices in fact gradually evolved through steps of a single amino acid residue towards achieving a certain optimal length for the delivery of free energy at a desired active site. For the remainder of the simulations, in order to ensure a sufficiently large arena with high spatial resolution of multi-soliton dynamics with $Q>1$, we will consider a protein $\alpha$-helix spine with a fixed length of $n_{\max }=40$ peptide groups. This length has been also used in our previous works [54, 86 and allows direct 
a

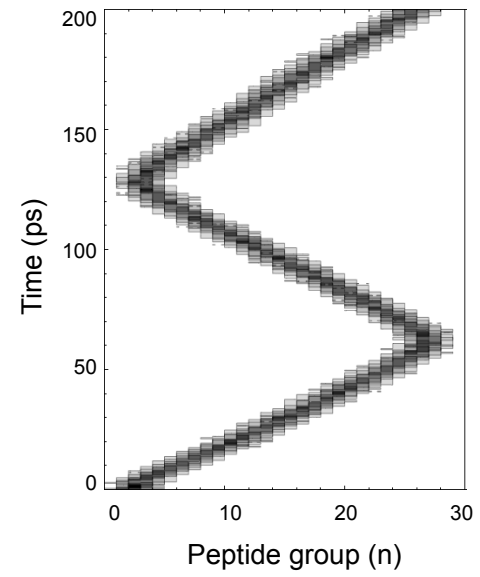

d

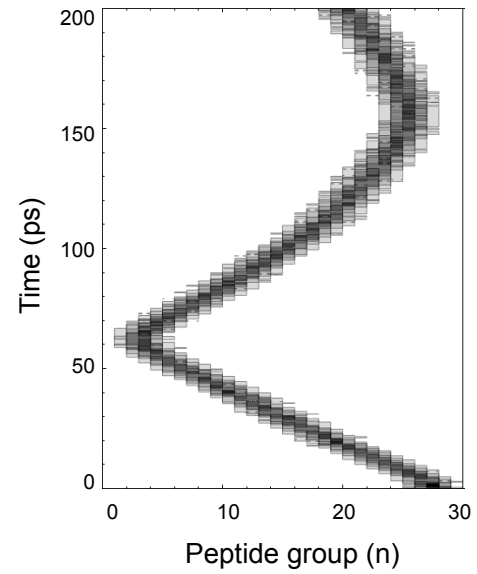

b

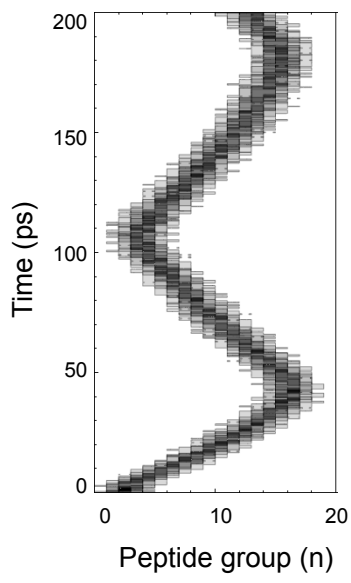

e

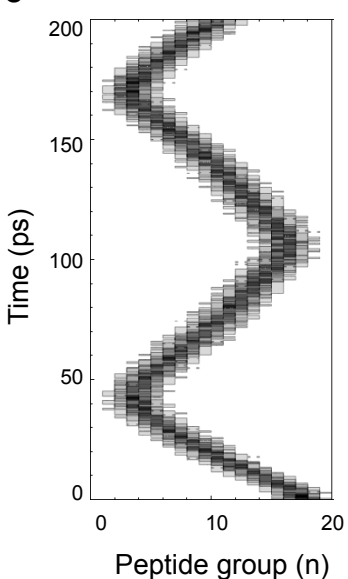

C

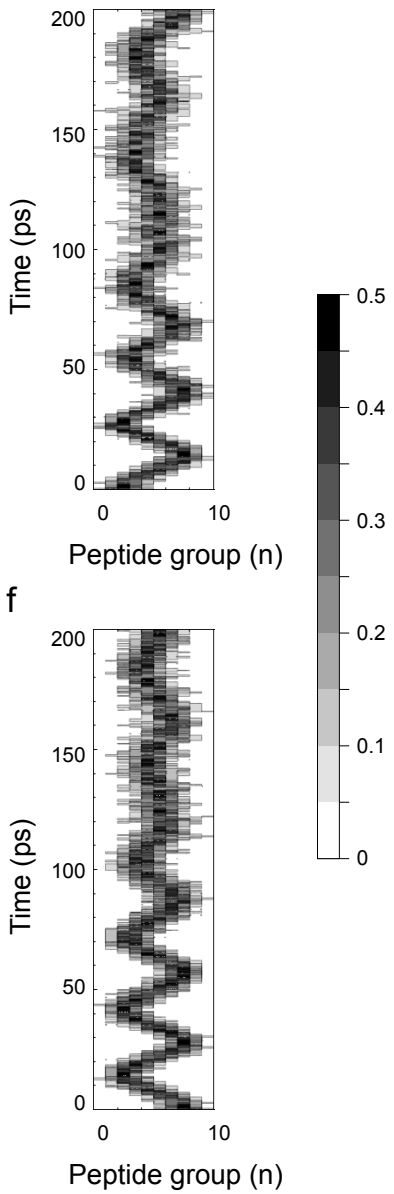

Figure 3: The quantum dynamics of a moving Davydov soliton for completely anisotropic exciton-phonon interaction $\xi=0$, with $Q=1$ quantum of amide I energy visualized through the expectation value of the exciton number operator $Q\left|a_{n}\right|^{2}$ at each peptide group $n$. (a-c) The Gaussian pulse of amide I energy is applied at the N-end of a short protein $\alpha$-helix spine with varying length, $n_{\max }=30$ (a), $n_{\max }=20$ (b) or $n_{\max }=10$ (c). (d-f) The Gaussian pulse of amide I energy is applied at the C-end of a short protein $\alpha$-helix spine with varying length, $n_{\max }=30$ (d), $n_{\max }=20$ (e) or $n_{\max }=10$ (f).

comparison of the newly obtained results with the data that has already been published.

\subsection{Multi-quanta solitons}

Previously we have shown that for $Q=1$, pulses of Gaussian amide I energy are able to launch traveling solitons when applied to the protein $\alpha$-helix ends, but generate pinned solitons if the amide I energy is applied in the interior of the protein $\alpha$-helix [54]. To study the collision of several Davydov solitons for $Q>1$, here we have considered a number of different scenarios in which the anisotropy of exciton-phonon interaction was systematically varied from $\xi=1$ to $\xi=0$ in steps of 0.1 . Because the quantum dynamics was affected nonlinearly with variation of $\xi$, we have presented four panels per simulation in such a way that the exhibited changes between any two consecutive panels are the most prominent. 
a

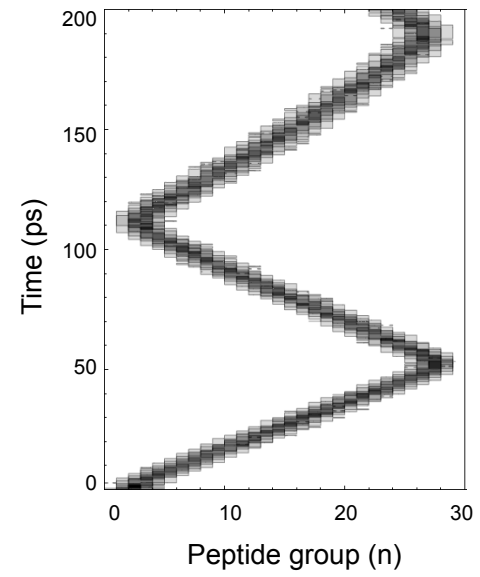

d

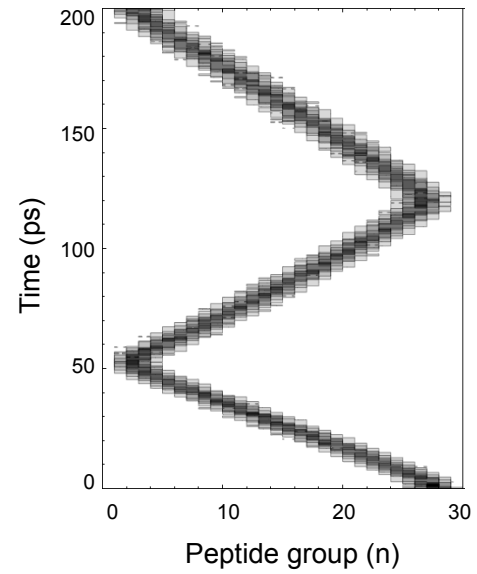

b

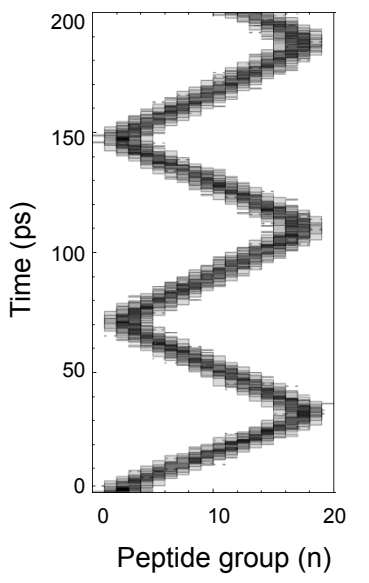

e

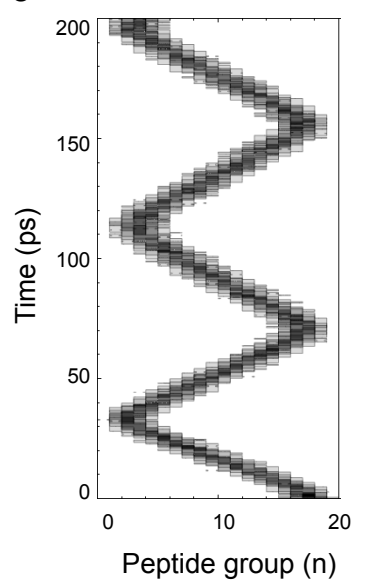

C

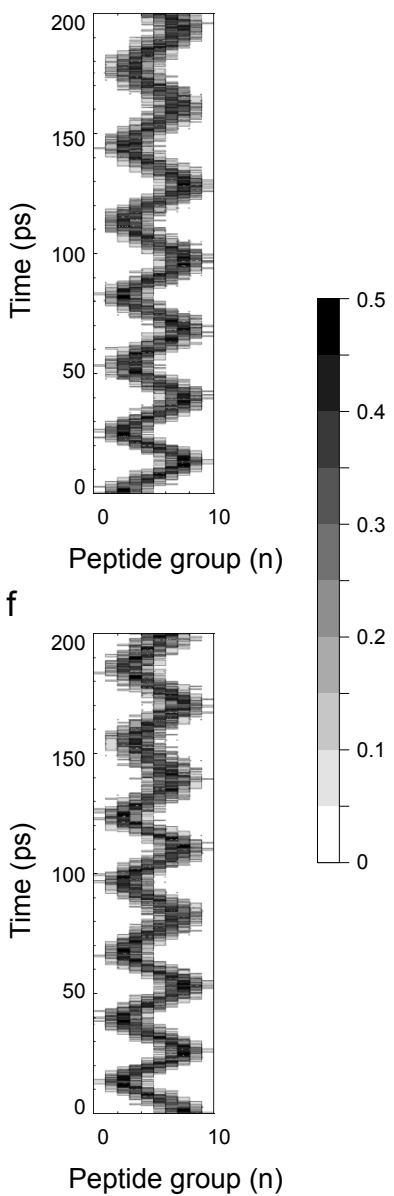

Figure 4: The quantum dynamics of a moving Davydov soliton for very slightly isotropic exciton-phonon interaction $\xi=0.02$, with $Q=1$ quantum of amide I energy visualized through the expectation value of the exciton number operator $Q\left|a_{n}\right|^{2}$ at each peptide group $n$. $(\mathrm{a}-\mathrm{c})$ Gaussian pulse of amide I energy is applied at the N-end of a short protein $\alpha$-helix spine with varying length, $n_{\max }=30$ (a), $n_{\max }=20$ (b) or $n_{\max }=10$ (c). (d-f) Gaussian pulse of amide I energy is applied at the C-end of a short protein $\alpha$-helix spine with varying length, $n_{\max }=30(\mathrm{~d}), n_{\max }=20(\mathrm{e})$ or $n_{\max }=10(\mathrm{f})$.

\subsubsection{Double solitons}

The simplest extension of the $Q=1$ case is to apply a single Gaussian of amide I energy over 5 peptide groups while exciting $Q=2$ amide I quanta. As noted by Kerr and Lomdahl [74], the multi-quantum property of the ansatz state (5) results in a stronger driving force on the phonon modes (34), but no modification of the equation for the amide I exciton probability amplitudes (33). Thus, it might be expected that double solitons may exhibit features similar to those resulting from an increased exciton-phonon coupling $\bar{\chi}$; namely, greater soliton stability, lower soliton velocity, and assisted soliton pinning [54]. Effectively, double Davydov solitons with $Q=2$ launched from the N-end of the protein $\alpha$-helix for completely isotropic exciton-phonon interaction $\xi=1$ moved at lower velocity of $147 \mathrm{~m} / \mathrm{s}$ (Fig. 5a) compared with velocity of $334 \mathrm{~m} / \mathrm{s}$ for $Q=1$ (Fig. 2a). Similarly, for completely anisotropic exciton-phonon interaction $\xi=0$ the velocity of the soliton with $Q=2$ was also lower, in fact zero due to soliton pinning (Fig. 5d), compared with velocity of $218 \mathrm{~m} / \mathrm{s}$ for 

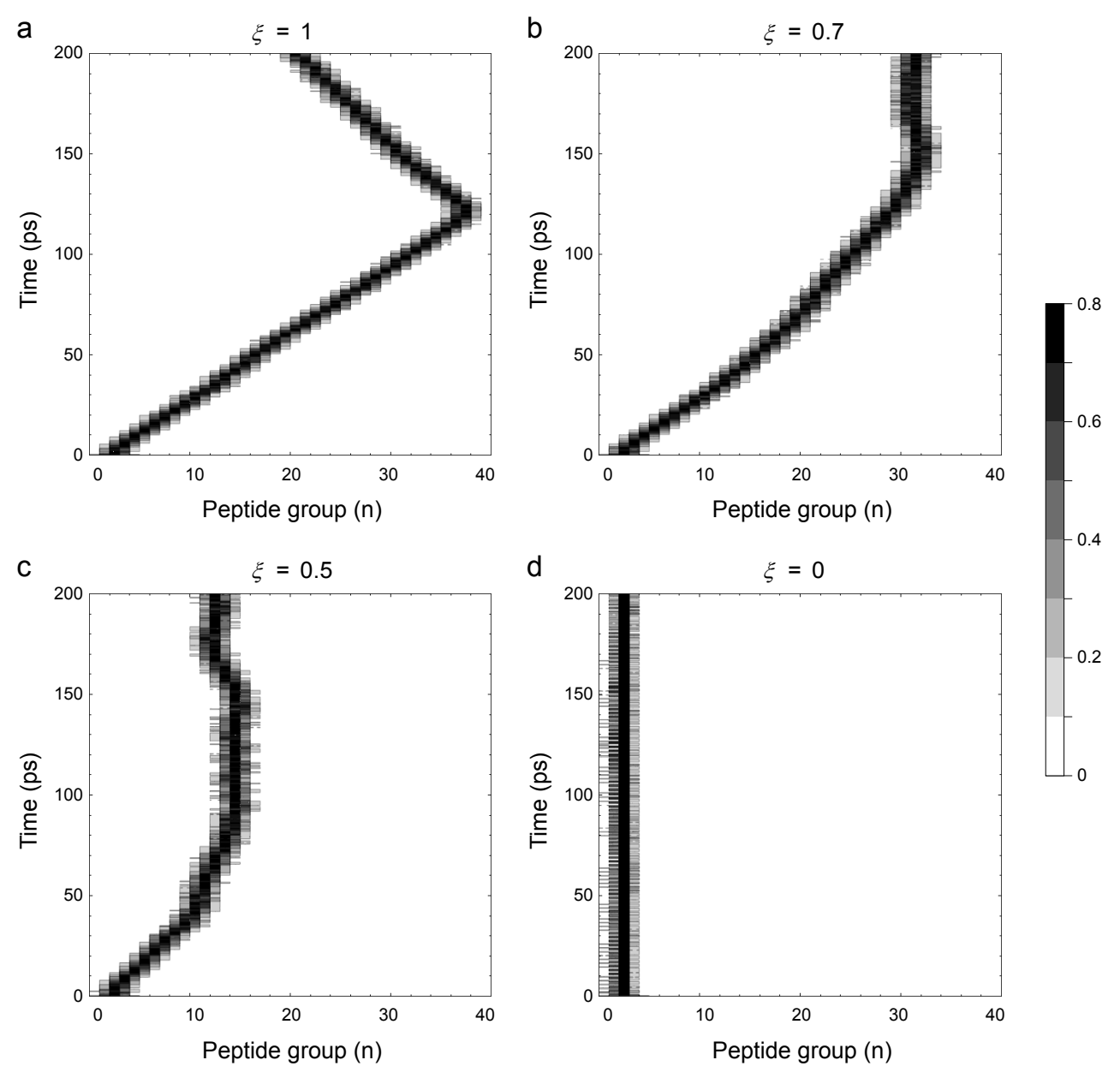

Figure 5: The quantum dynamics of a double Davydov soliton with $Q=2$ quanta of amide I energy delivered as a single Gaussian pulse over 5 peptide groups at the N-end of a protein $\alpha$-helix visualized through the expectation value of the exciton number operator $Q\left|a_{n}\right|^{2}$. Decreasing the isotropy of exciton-phonon interaction leads to reduction in soliton velocity and eventual soliton pinning: $\xi=1$ (a), $\xi=0.7$ (b), $\xi=0.5$ (c) and $\xi=0$ (d).

$Q=1$ (Fig. 3a). For intermediate values of $\xi$, the soliton migrated towards the interior of the protein $\alpha$-helix where it started wobbling around some fixed interior point (Figs. $5 \mathrm{~b}, \mathrm{c}$ ).

When the double soliton was generated in the interior of the protein $\alpha$-helix, it was pinned and wobbled around its initial position (Fig. 6). When the isotropy of exciton-phonon interaction $\xi$ was decreased, this led to reduction in the wobbling of the pinned soliton, which eventually came to a complete halt for $\xi=0$ (Fig. 6d). Thus, increasing the number of amide I quanta increases the energy of the soliton, enhances soliton stability and in turn lowers the soliton propagation velocity.

\subsubsection{Two-soliton collisions}

The presence of multiple amide I quanta in the protein $\alpha$-helix allows for the application of an initial multi-Gaussian distribution, which is a sum of several Gaussians, in order to generate several solitons whose eventual collision may lead to detecting either constructive, or destructive, quantum interference phenomena. This could be instrumental in how proteins utilize free energy for driving life-supporting bio-processes. 

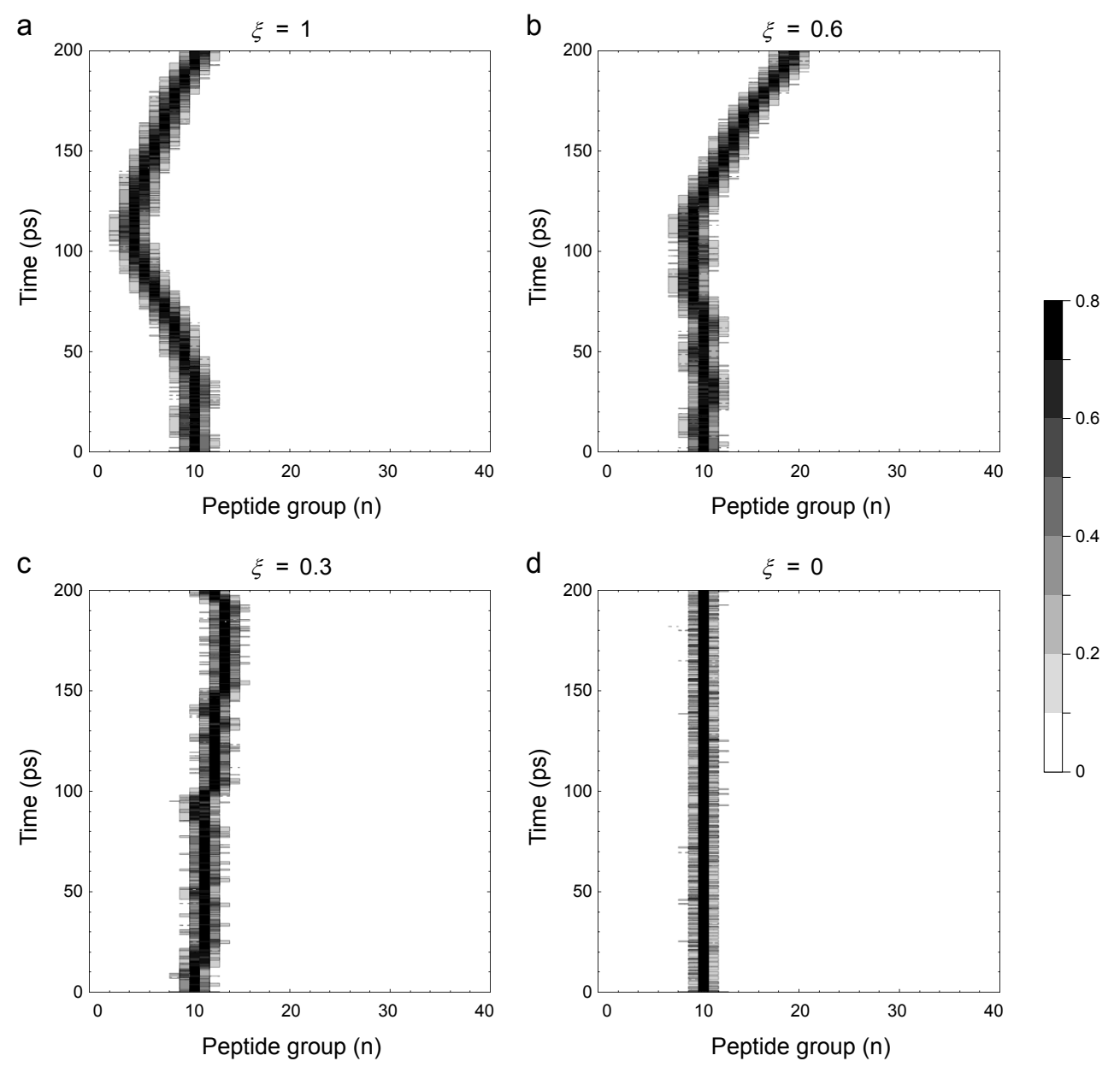

Figure 6: The quantum dynamics of a double Davydov soliton with $Q=2$ quanta of amide I energy delivered as a single Gaussian pulse over 5 peptide groups $n=9-13$ in the interior of a protein $\alpha$-helix visualized through the expectation value of the exciton number operator $Q\left|a_{n}\right|^{2}$. Decreasing the isotropy of exciton-phonon interaction leads to reduction in wobbling of the pinned soliton: $\xi=1$ (a), $\xi=0.6$ (b), $\xi=0.3$ (c) and $\xi=0$ (d).

The launching of two propagating solitons from the two ends of the protein $\alpha$-helix, for $\xi=1$, leads to significant constructive quantum interference of amide I quantum probability amplitudes $a_{n}$, which focuses the amide I energy at the collision site to a width much narrower than each of the individual solitons (Fig. 7 a). Thus, constructive quantum interference may provide a mechanism for the brief focusing of energy at protein active centers for catalysis of biologically important reactions.

Interestingly, increasing the anisotropy of exciton-phonon interaction by lowering $\xi<1$, is capable of inducing destructive quantum interference at sites of soliton collision, so that these solitons appear to bounce off each other without even touching (Fig. 7b). For $\xi<0.2$, the soliton collision may lead to pinning of the soliton, which may wobble out of the collision site for $\xi=0.1$ (Fig. 7 k) or remain pinned at the collision site for $\xi=0$ (Fig. $7 \mathrm{~d}$ ). Thus, another potentially useful mechanism for persistent pinning of energy at protein active centers may be the local modification of exciton-phonon interaction anisotropy towards lower $\xi$ values.

Launching a propagating soliton from one end of the protein $\alpha$-helix towards a pinned soliton in the interior of the helix, for $\xi=1$, leads to destructive quantum interference of amide I quantum probability amplitudes $a_{n}$ at the collision site with the moving and pinned 

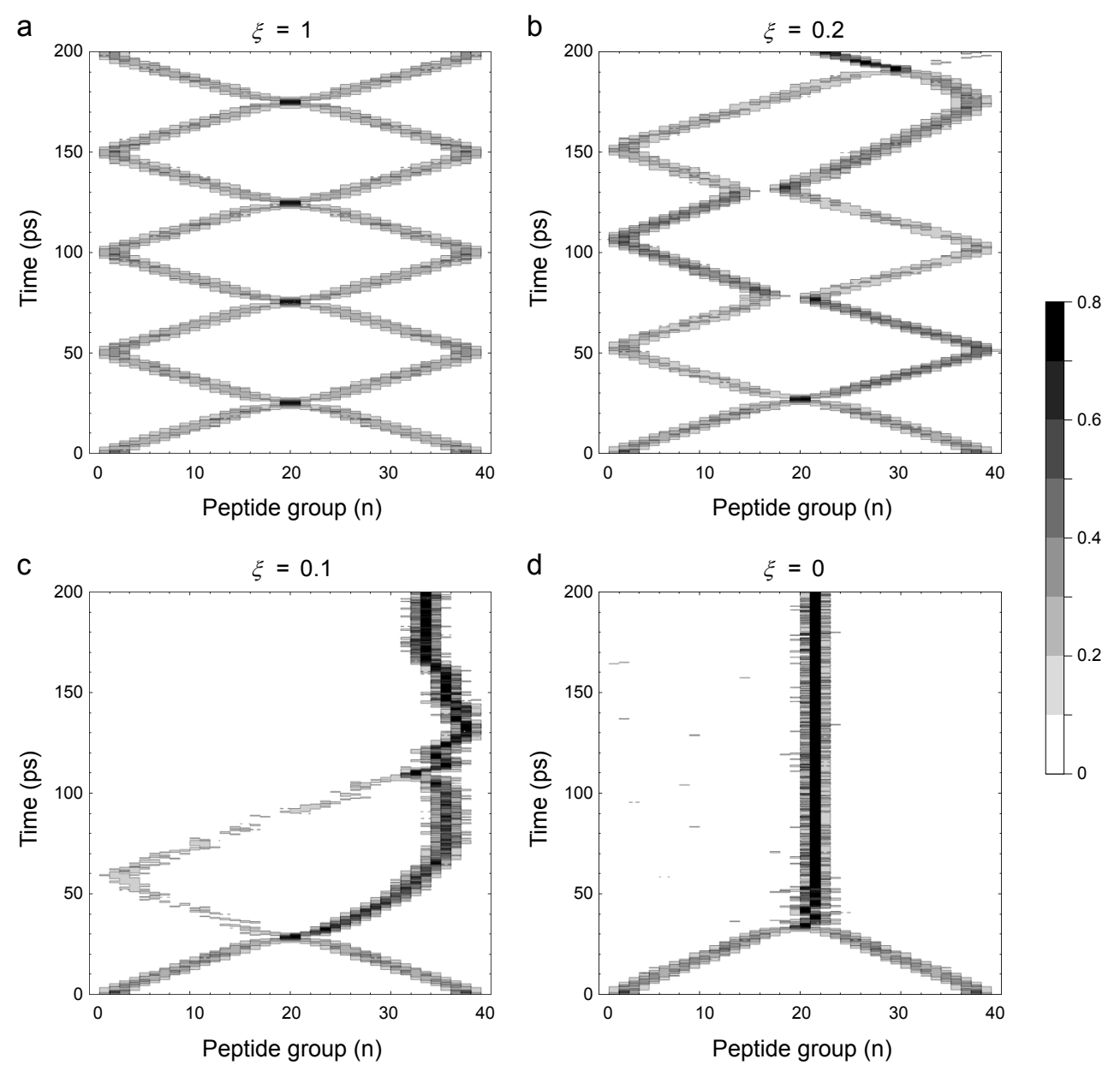

Figure 7: The quantum collision of two moving Davydov solitons with $Q=2$ quanta of amide I energy launched from the two ends of a protein $\alpha$-helix visualized through the expectation value of the exciton number operator $Q\left|a_{n}\right|^{2}$. Constructive quantum interference may focus the amide I energy at the collision site to a width much narrower than each of the individual solitons. Decreasing the isotropy of exciton-phonon interaction may result in soliton pinning after the collision: $\xi=1$ (a), $\xi=0.2(\mathrm{~b}), \xi=0.1$ (c) and $\xi=0$ (d).

soliton switching their roles between collisions (Fig. 83). In such collision scenario, lowering of the isotropy of exciton-phonon interaction through the parameter $\xi$ leads to replacement of destructive with constructive quantum interference at the collision sites (Figs. 8b,c). For $\xi=0$, the collision of the two solitons at a site with constructive quantum interference again leads to pinned soliton (Fig. 8d). The irregular trajectories of solitons in the computer simulations highlight the nonlinear dependence of the observed quantum dynamics on the boundary conditions and point towards the emergence of quantum chaos in regard to dynamics of quantum expectation values of amide I energy.

\subsubsection{Three-soliton collisions}

Increasing the number of amide I quanta to $Q=3$ allows for collision of two moving solitons with one pinned soliton at a central position (Fig. 9) or at a non-central position (Fig. 10). In both of these cases, the pinned soliton appears to act as a divider, which splits the protein $\alpha$-helix into compartments. The two moving solitons, one launched from the N-end and the 

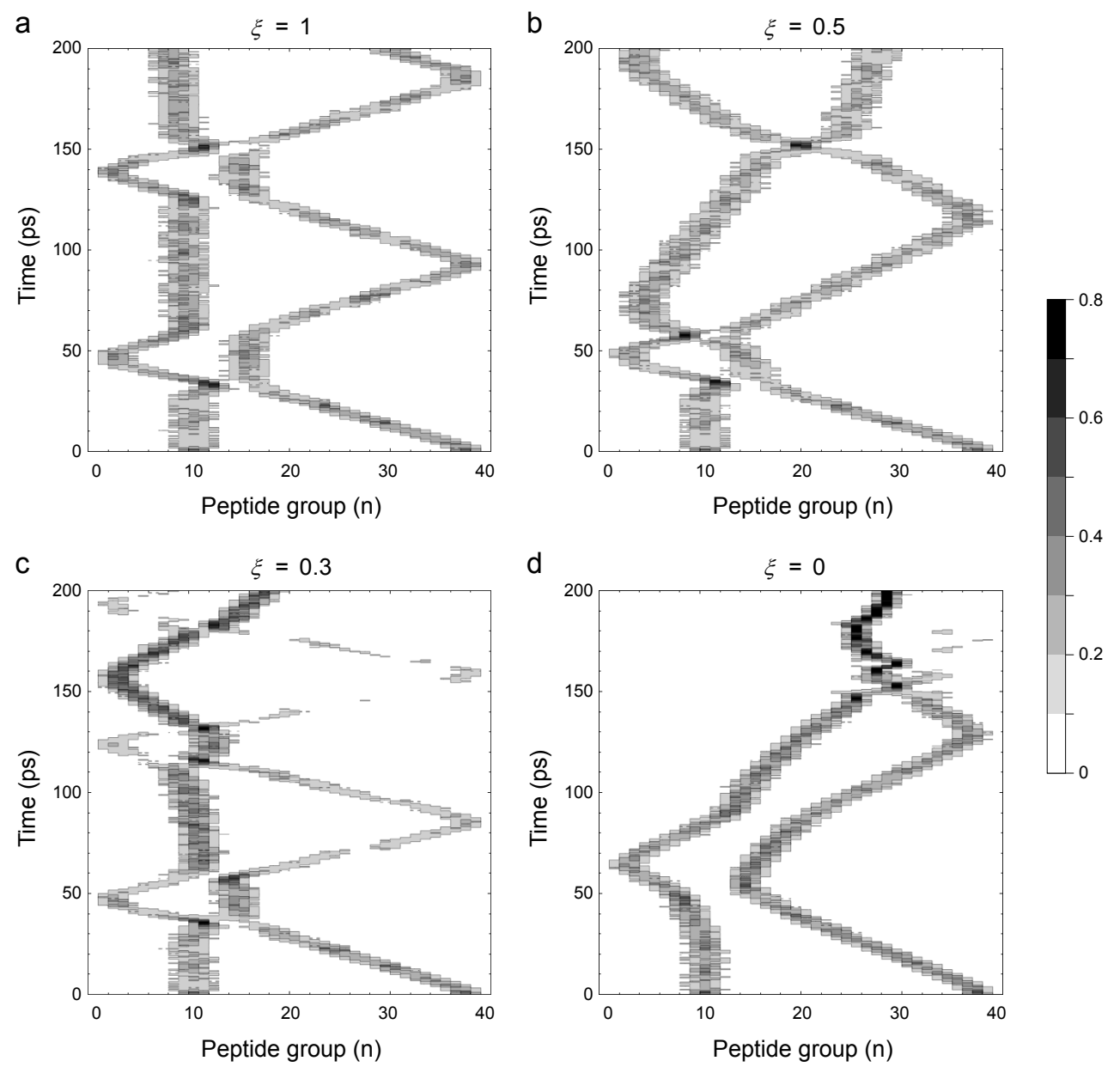

Figure 8: The quantum collision of one pinned and one moving Davydov soliton with $Q=2$ quanta of amide I energy visualized through the expectation value of the exciton number operator $Q\left|a_{n}\right|^{2}$. Decreasing the isotropy of exciton-phonon interaction leads to soliton pinning after the collision: $\xi=1$ (a), $\xi=0.5$ (b), $\xi=0.3$ (c) and $\xi=0$ (d).

other launched from the C-end, then reflect forth and back within the left compartment or the right compartment, respectively (Fig. 9 ). Because the soliton width is spread over 5 peptide groups, which is an odd number, in the simulations with central soliton the protein length was set to $n_{\max }=41$ peptide groups in order to be able to perfectly center the soliton. Decreasing the isotropy of exciton-phonon interaction by lowering $\xi<1$ introduces asymmetry in the quantum dynamics with accidental drifts to the right (Fig. 9b) or to the left (Figs. 95,d) of the central pinned soliton and renders irregular the trajectories of the two propagating solitons (Figs. 9b-d). For $\xi \leq 0.1$, constructive quantum interference gives birth to a pinned soliton at the site of collision (Fig. 9k,d) qualitatively reproducing the behavior observed in two-soliton collisions (Figs. $7 \mathrm{~d}$ and $8 \mathrm{~d}$ ).

The three-soliton collision between two moving solitons and one non-central pinned soliton exhibited even richer quantum dynamics (Fig. 10). In such scenario, for $\xi=1$ the moving soliton launched from the N-end of the protein $\alpha$-helix collided first with the pinned soliton at $n=11-15$ switching the roles of the moving and pinned soliton similarly to the twosoliton collision (cf. Fig. 10a vs Fig. 8a), whereas the moving soliton launched from the C-end remained compartmentalized and reflected forth and back without actual collision (cf. Fig. 10a vs Fig. 9a). Again, decreasing the isotropy of exciton-phonon interaction by lowering $\xi<1$ 

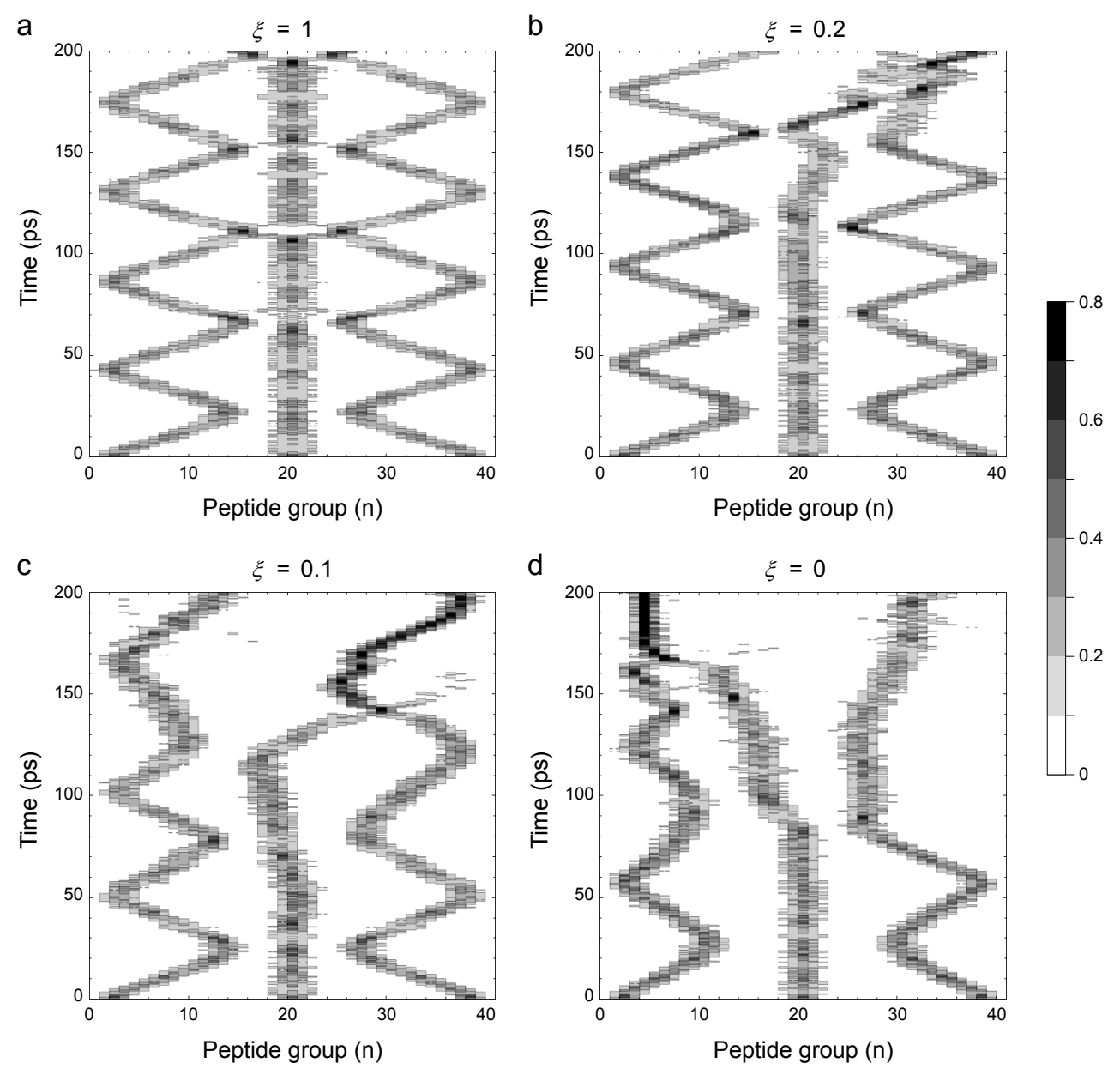

Figure 9: The quantum collision of one central pinned soliton and two moving Davydov solitons with $Q=3$ quanta of amide I energy visualized through the expectation value of the exciton number operator $Q\left|a_{n}\right|^{2}$. Destructive quantum interference prevents the solitons from colliding and it appears that they repel each other. Decreasing the isotropy of exciton-phonon interaction may lead to soliton pinning after the collision: $\xi=1$ (a), $\xi=0.2$ (b), $\xi=0.1$ (c) and $\xi=0(\mathrm{~d})$.

led to irregularities in the soliton trajectories (Figs. 10, d). For the completely anisotropic exciton-phonon interaction $\xi=0$, the three solitons collided in the center of the protein $\alpha$-helix producing a pinned soliton that did not wobble around (Fig. 10 d).

To further test the effect on compartmentalization of the protein $\alpha$-helix by a central pinned soliton, we have doubled the central soliton raising the total number of amide I quanta to $Q=4$ (Fig. 11). Interestingly, for $\xi=1$ the double central soliton stayed in the center where it devoured the single solitons feeding on their quantum probability amplitudes (Fig. 11a). Decreasing the isotropy of exciton-phonon interaction by lowering $\xi<1$ introduced irregular wobbling of the central soliton (Figs. 11p-d). Thus, the collisions between single and double solitons in protein $\alpha$-helices may have detrimental effects upon single solitons.

\subsection{Quantum tunneling of solitons}

Having verified the occurrence of quantum interference in soliton collisions, we have turned our attention to the possibility of quantum tunneling through massive barriers. Because the 

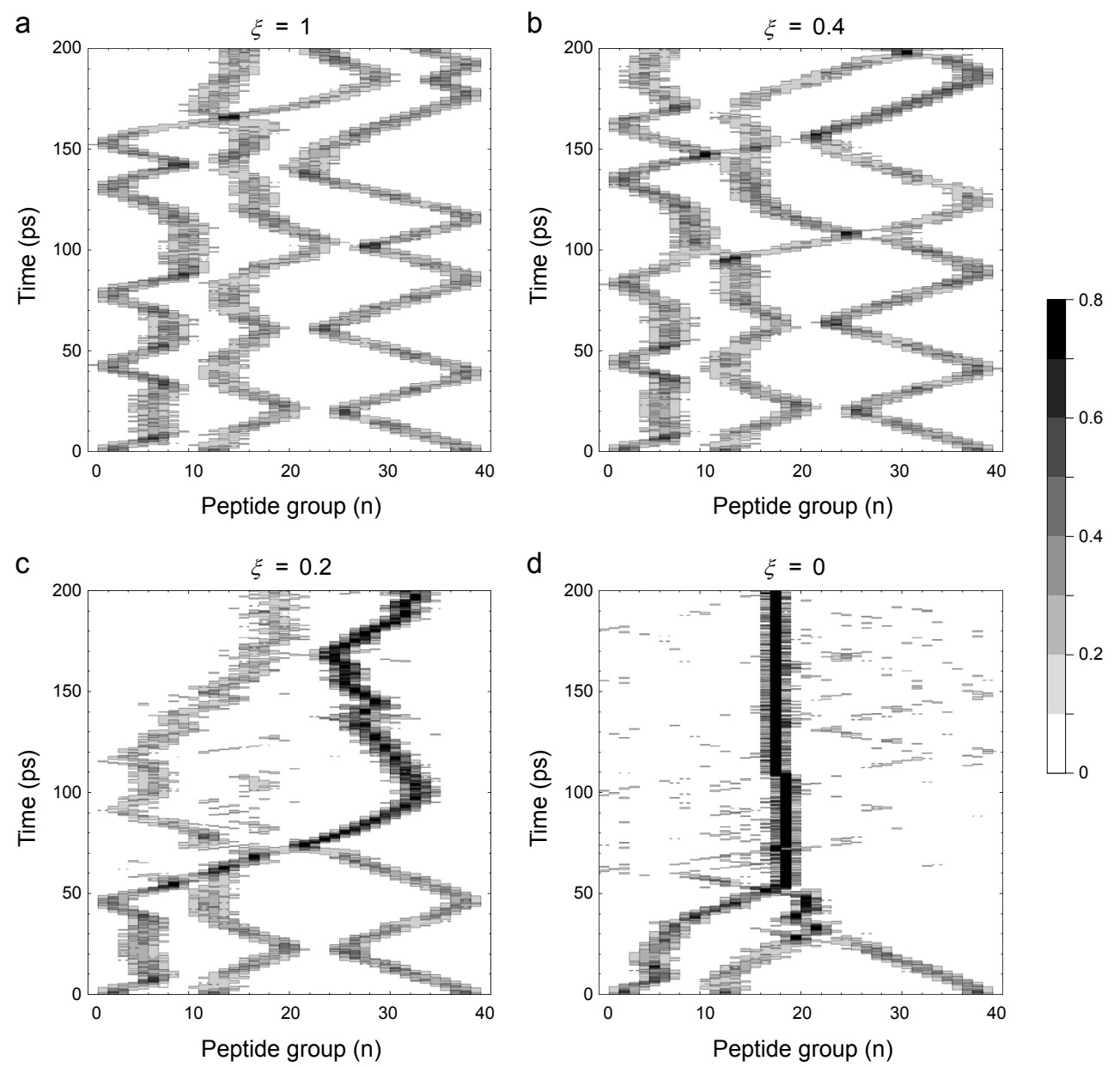

Figure 10: The quantum collision of one non-central pinned soliton and two moving Davydov solitons with $Q=3$ quanta of amide I energy visualized through the expectation value of the exciton number operator $Q\left|a_{n}\right|^{2}$. Destructive quantum interference prevents the solitons from colliding and it appears that they repel each other. Decreasing the isotropy of exciton-phonon interaction may lead to soliton pinning after the collision: $\xi=1$ (a), $\xi=0.4$ (b), $\xi=0.2$ (c) and $\xi=0(\mathrm{~d})$.

second of Davydov equations (34) depends on the mass of individual peptide groups $M_{n}$, in our previous work [86] we have studied whether external protein clamps could act as massive barriers by raising locally the effective mass of peptide groups inside a protein $\alpha$-helix. In this latter case, we have shown that single solitons with $Q=1$ that are wider behave as quasiparticles with higher energy and are capable of tunneling through heavier barriers in comparison with narrower solitons [86].

Here, we have investigated the effect of increasing the number of amide I quanta for a fixed soliton width. To set a base for comparison, we have first launched a single Davydov soliton with $Q=1$ by a Gaussian pulse of amide I energy distributed over 5 peptide groups at the $\mathrm{N}$-end of the protein $\alpha$-helix and aimed it at a massive barrier located over three peptide groups $n=26-28$. This was repeated for the two limiting cases, $\xi=1$ and $\xi=0$, of exciton-phonon interaction isotropy (Figs. 12 and 13 .

For $\xi=1$, the soliton readily tunneled through the barrier in which each of the three peptide groups was with effective mass of $200 M$ (Fig. 12 b), but got reflected from heavier barriers with $400 M$ (Fig. 12k) or $600 M$ (Fig. $12 \mathrm{~d}$ ). Thus, as it may be expected, increasing 

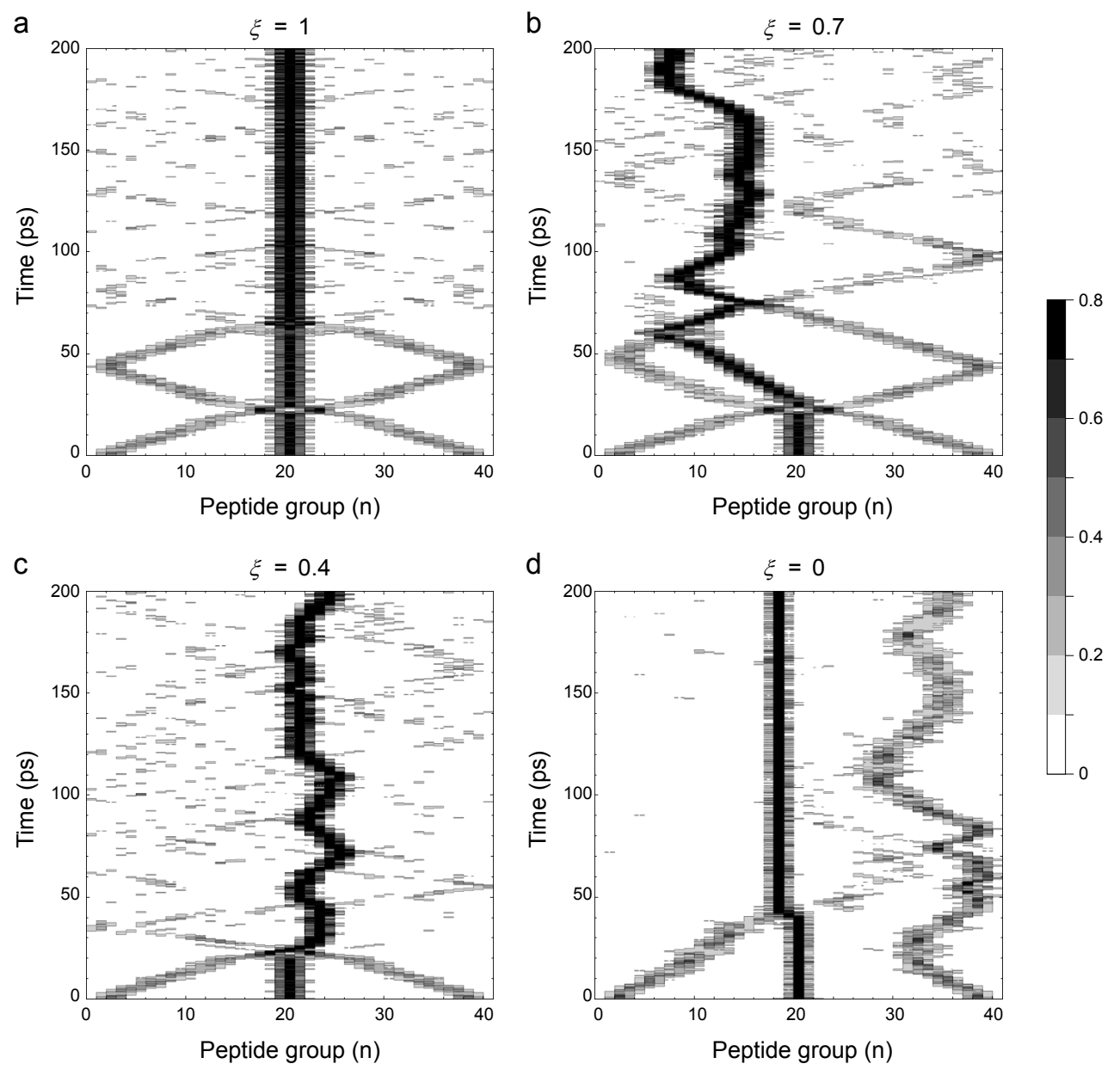

Figure 11: The quantum collision of one double central pinned soliton and two moving Davydov solitons with $Q=4$ quanta of amide I energy visualized through the expectation value of the exciton number operator $Q\left|a_{n}\right|^{2}$. Decreasing the isotropy of exciton-phonon interaction leads to reduction in the shift induced by the collision and suppresses the wobbling of the pinned soliton: $\xi=1$ (a), $\xi=0.7$ (b), $\xi=0.4$ (c) and $\xi=0$ (d).

the mass of the barrier acts analogously to increasing the height of a potential barrier thereby reducing the probability of quantum tunneling of the soliton and increasing the probability of its reflection.

For $\xi=0$, the soliton dynamics is not mirror symmetric with respect to launching from the N-end or the C-end of the protein $\alpha$-helix. Despite the lack of complete mirror symmetry, qualitatively the soliton behavior was similar: it was able to tunnel through both $200 \mathrm{M}$ barrier (Figs. 13b and 14b) and $400 M$ barrier (Figs. 13p and 14 ), but reflected from the $600 M$ barrier (Figs. $13 \mathrm{~d}$ and $14 \mathrm{~d}$ ). The soliton tunneling time through $200 M$ barrier was also faster for $\xi=0,22.4 \mathrm{ps}$ when launched from the N-end (Fig. 13b) and $29.8 \mathrm{ps}$ when launched from the C-end (Fig. 14p), compared with $33.2 \mathrm{ps}$ for $\xi=1$ (Fig. 12b), which is the same for launching from either end of the $\alpha$-helix. Thus, consistently with our previously reported results for $Q=1[86$ decreasing the isotropy of exciton-phonon interaction by lowering $\xi$, decreases the probability of soliton reflection from the barrier, increases the probability of quantum tunneling of the soliton through the barrier and reduces the tunneling time in the event of successful tunneling.

It should be noted that the soliton width does not appear to be significantly affected by 

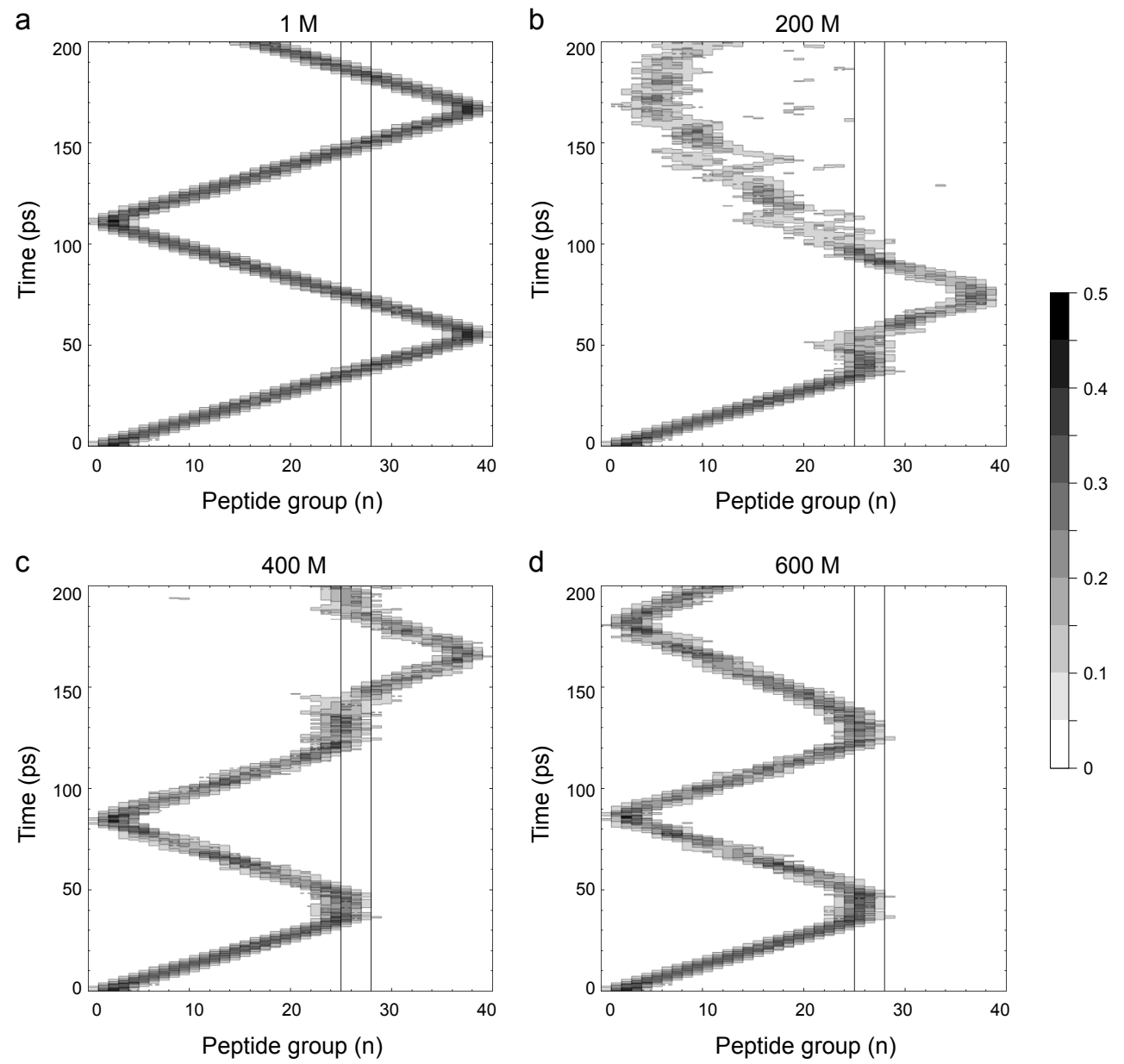

Figure 12: The quantum dynamics of a Davydov soliton with $Q=1$ quantum of amide I energy tunneling through or reflecting from a massive barrier located over three peptide groups $n=26-28$ visualized through the expectation value of the exciton number operator $Q\left|a_{n}\right|^{2}$ for $\xi=1$. Increasing the mass of the barrier decreases the probability of tunneling and increases the probability of reflection: no barrier $1 M(\mathrm{a})$, barrier in which each of the three peptide groups is with effective mass of $200 M$ (b), $400 M$ (c) and $600 M$ (d). The barrier location is indicated with thin vertical lines.

the anisotropy of exciton-phonon interaction with $\xi<1$. The soliton width, defined by the spread of the exciton quantum probability amplitudes, is directly related to the expectation value of the exciton energy operator

$$
\left\langle\Psi\left|\hat{H}_{\mathrm{ex}}\right| \Psi\right\rangle=Q \sum_{n}\left[E_{0}\left|a_{n}\right|^{2}-J_{n+1} a_{n}^{*} a_{n+1}-J_{n} a_{n}^{*} a_{n-1}\right]
$$

The gauge transformation $a_{n} \rightarrow \bar{a}_{n} e^{-\frac{\imath}{\hbar} \int \gamma(t) d t}$ used to remove the highly oscillatory phase in Davydov's equations effectively sets $E_{0}=0$. For the case when all $J_{n}$ are equal, the expectation of the exciton energy operator becomes

$$
\left\langle\Psi\left|\hat{H}_{\mathrm{ex}}\right| \Psi\right\rangle=-2 Q J \sum_{n}\left[\operatorname{Re}\left(a_{n}\right) \operatorname{Re}\left(a_{n+1}\right)+\operatorname{Im}\left(a_{n}\right) \operatorname{Im}\left(a_{n+1}\right)\right]
$$

Thus, the soliton width is positively related to the absolute value of the exciton expectation energy. When the soliton is narrowly focused onto a single peptide group, then the expectation 

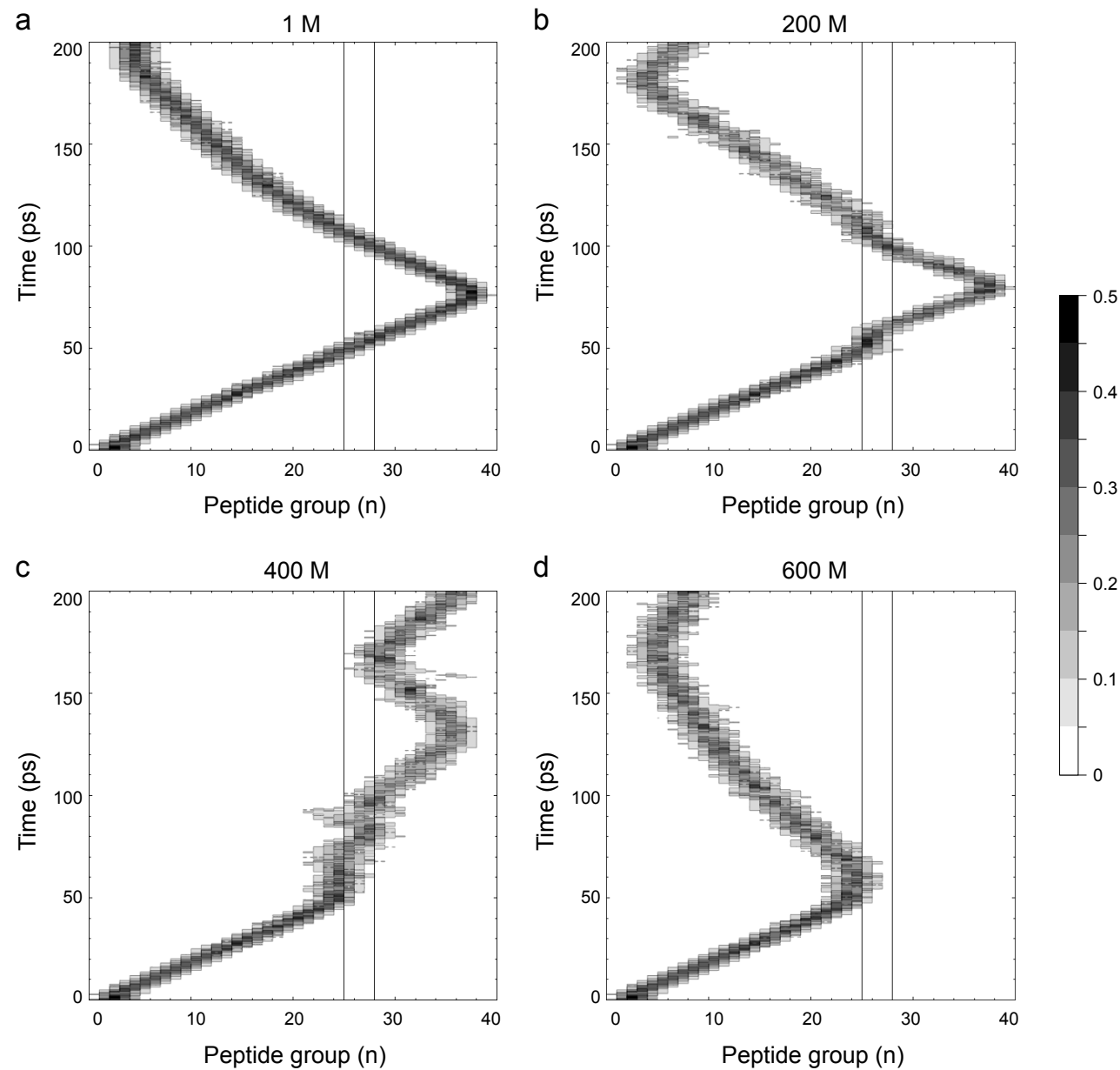

Figure 13: The quantum dynamics of a Davydov soliton with $Q=1$ quantum of amide I energy tunneling through or reflecting from a massive barrier located over three peptide groups $n=26-28$ visualized through the expectation value of the exciton number operator $Q\left|a_{n}\right|^{2}$ for $\xi=0$. Increasing the mass of the barrier decreases the probability of tunneling and increases the probability of reflection: no barrier $1 M(\mathrm{a})$, barrier in which each of the three peptide groups is with effective mass of $200 M$ (b), $400 M$ (c) and $600 M$ (d). The barrier location is indicated with thin vertical lines.

value is minimal $\left|\left\langle\Psi\left|\hat{H}_{\text {ex }}\right| \Psi\right\rangle\right|=0$, whereas when the soliton is evenly spread over all peptide groups in the $\alpha$-helix spine the expectation value is maximal $\left|\left\langle\Psi\left|\hat{H}_{\text {ex }}\right| \Psi\right\rangle\right|=2 Q J$.

To correctly determine the soliton width in the discrete lattice, it is necessary to consider the fact that, for most of the time, the soliton is in the process of transition between neighboring peptide groups. Thus, one would need a methodological rule that identifies time points when the soliton is best positioned over the peptide groups for measuring its width. Furthermore, on top of the soliton-induced deformation of the lattice of hydrogen bonds there are superposed small disturbances due to the phonon oscillations of the lattice, which may introduce some noise on the exciton envelope of the soliton. Therefore, to find out whether the soliton width changes in the course of the whole simulated time period of $200 \mathrm{ps}$, we have divided the $\alpha$-helix spine into 35 overlapping local stretches of excition expectation energy 

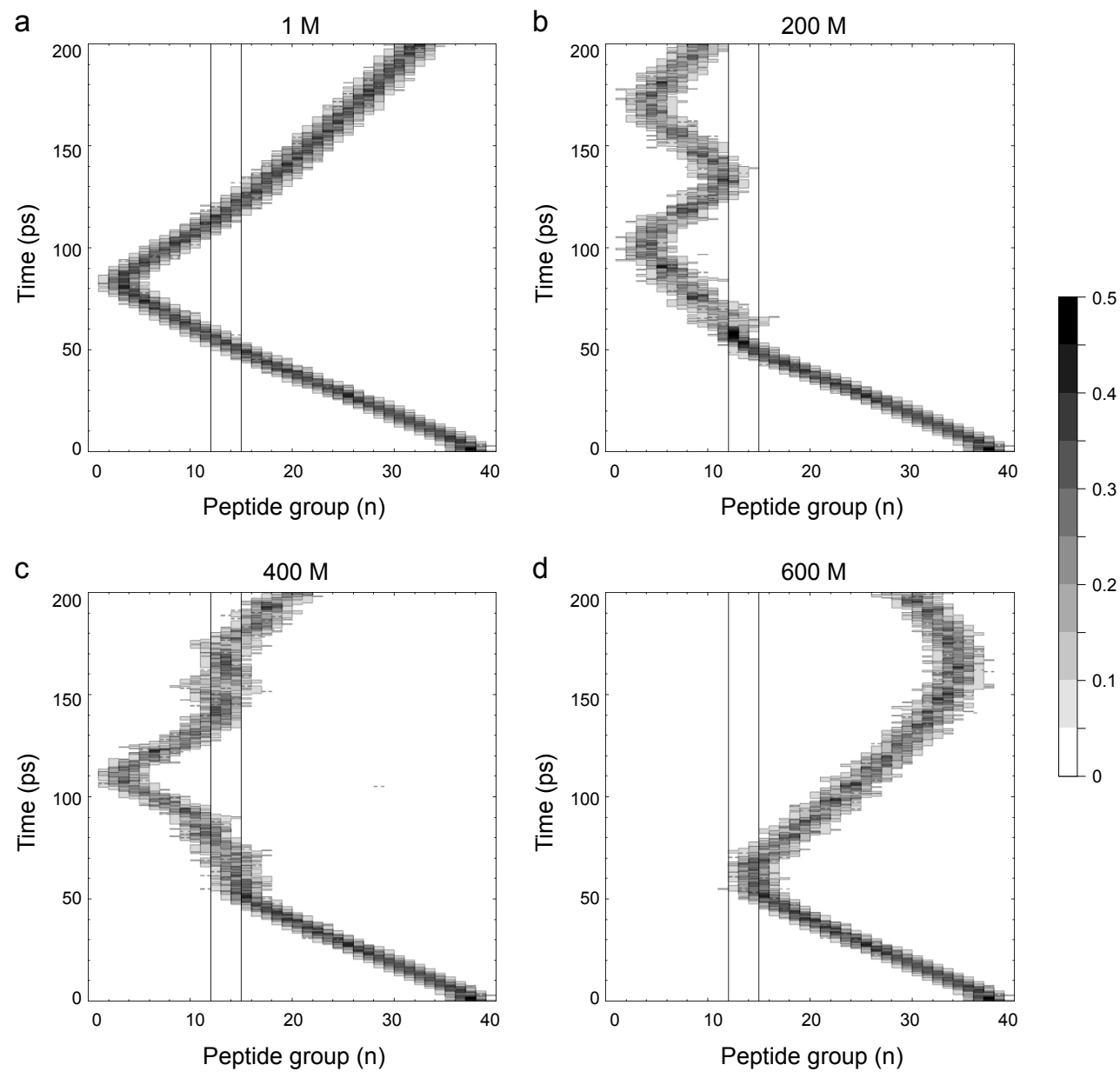

Figure 14: The quantum dynamics of a Davydov soliton with $Q=1$ quantum of amide I energy launched from the $\mathrm{C}$-end of the $\alpha$-helix tunneling through or reflecting from a massive barrier located over three peptide groups $n=13-15$ visualized through the expectation value of the exciton number operator $Q\left|a_{n}\right|^{2}$ for $\xi=0$. Increasing the mass of the barrier decreases the probability of tunneling and increases the probability of reflection: no barrier $1 M$ (a), barrier in which each of the three peptide groups is with effective mass of $200 M$ (b), $400 M$ (c) and $600 M(\mathrm{~d})$. The barrier location is indicated with thin vertical lines.

given by 5 terms in the sum for $\left|\left\langle\Psi\left|\hat{H}_{\text {ex }}\right| \Psi\right\rangle\right|$ as follows

$$
\mathcal{E}_{i}(t)=2 Q J \sum_{n=i-2}^{i+2}\left[\operatorname{Re}\left(a_{n}\right) \operatorname{Re}\left(a_{n+1}\right)+\operatorname{Im}\left(a_{n}\right) \operatorname{Im}\left(a_{n+1}\right)\right]
$$

The choice of $i \in[3, \ldots, 37]$ ensures that the local stretches do not extend outside the $\alpha$-helix spine ends, whereas the sum over 5 terms ensures that exciton probability amplitudes over 6 peptide groups are captured. Then, the motion of the soliton leads to sequential peaks of neighboring $\mathcal{E}_{i}(t)$ separated by the time period needed for the soliton to travel from a position perfectly centered on $\mathcal{E}_{i}(t)$, to a spatially translated position when the soliton is centered on $\mathcal{E}_{i+1}(t)$. Variation of the time intervals between peaks of neighboring local stretches $\mathcal{E}_{i}(t)$ indicates a variation in the soliton speed, whereas a non-zero slope of the trend line of exciton energy peaks will be an indication of changing soliton width. For example, a positive slope of the trend line of exciton energy peaks will indicate that the soliton becomes wider, whereas 
a negative trend line of exciton energy peaks will indicate that the soliton becomes narrower. This will hold true as long as the soliton width fits inside the stretches $\mathcal{E}_{i}(t)$ (hence no contributions to the exciton expectation energy will be trimmed) and explains why we have set the length of the streches to be 6 peptide groups given that the initial soliton width is 5 peptide groups.

To assess the effects of $\xi$ on soliton width and velocity, we have compared the simulations with $Q=1$ reported in Figs. $12 \mathrm{a}$, 13 and 14 ar $\xi=1$ launched from the N-end, and for $\xi=0$ launched from the N-end or C-end, respectively. The distances between consecutive $\mathcal{E}_{i}(t)$ peaks are shorter for $\xi=1$ compared with $\xi=0$ (Figs. 15a,c,e) consistent with higher velocities of $\xi=1$ solitons. While the soliton velocity is relatively constant for $\xi=1$ for the whole simulation period of $200 \mathrm{ps}$ (Fig. 15b), it appears to slow down for $t>120 \mathrm{ps}$ for $\xi=0$ (Figs. 15d,f). This retardation of the $\xi=0$ solitons, however, is not accompanied by any significant spread in the envelope of exciton quantum probability amplitudes, since the trend lines for $\mathcal{E}_{i}(t)$ peaks remain horizontal at $1.6 J$ (Figs. 15d,f). Thus, the mechanism behind the varying soliton velocity for $\xi=0$ could be quantum interference within the finite length of the discrete lattice. Also, the ambient noise on the exciton envelope, resulting from the phonon lattice oscillations, is greater for $\xi=0$, and is probably due to manifestly nonlinear effects dependent on $\chi_{r}$, because to achieve the average $\bar{\chi}=35 \mathrm{pN}$, the right exciton-phonon coupling becomes $\chi_{r}=70 \mathrm{pN}$ given that $\chi_{l}=0 \mathrm{pN}$. In contrast, for $\xi=1$ the average $\bar{\chi}=35 \mathrm{pN}$ is obtained with $\chi_{r}=35 \mathrm{pN}$ and $\chi_{l}=35 \mathrm{pN}$.

Doubling the launched soliton by increasing the amide I quanta to $Q=2$, for $\xi=1$, reveals a soliton reflection from $200 M$ barrier (Fig. 16b) or $400 M$ barrier (Fig. 16 ), but remarkable tunneling phenomena through the much more massive $600 M$ barrier (Fig. $16 \mathrm{~d}$ ). This quantum behavior provides an indication that the tunneling of the Davydov soliton through the massive barrier could be analogous to a massive quantum particle tunneling through a potential barrier whose potential barrier height $V_{0}$ is lower than the energy $E_{0}$ of the particle 95,96 .

For a massive quantum particle with mass $m$ and energy $E_{0}$ tunneling through rectangular potential barrier

$$
V(x)=V_{0}\left[\Theta\left(x-x_{1}\right)-\Theta\left(x-x_{2}\right)\right]
$$

with height $V_{0}$ and width $\Delta x=x_{2}-x_{1}$, where $\Theta(x)=\frac{d}{d x} \max \{x, 0\}$ is the Heaviside step function, the transmission coefficient $T$ is determined from different quantum mechanical expressions depending on the magnitude of $V_{0}$ with respect to $E_{0}$, which is required to avoid the appearance of imaginary wavenumber $k_{2}$ as follows.

Case I: If $V_{0}>E_{0}$, on setting $\hbar k_{1}=\sqrt{2 m E_{0}}$ and $\hbar k_{2}=\sqrt{2 m\left(V_{0}-E_{0}\right)}$ [95, p. $\left.75-80\right]$, the analytic derivation of the transmission coefficient gives

$$
T=\frac{4 k_{1}^{2} k_{2}^{2}}{\left(k_{1}^{2}+k_{2}^{2}\right)^{2} \sinh ^{2}\left(\Delta x k_{2}\right)+4 k_{1}^{2} k_{2}^{2}}
$$

Case II: If $V_{0}<E_{0}$, on setting $\hbar k_{1}=\sqrt{2 m E_{0}}$ and $\hbar k_{2}=\sqrt{2 m\left(E_{0}-V_{0}\right)}[95$, p. $75-80]$, the analytic derivation of the transmission coefficient gives

$$
T=\frac{4 k_{1}^{2} k_{2}^{2}}{\left(k_{1}^{2}-k_{2}^{2}\right)^{2} \sin ^{2}\left(\Delta x k_{2}\right)+4 k_{1}^{2} k_{2}^{2}}
$$

In the latter case, for $\Delta x>\frac{1}{k_{2}}$, it is indeed possible to observe larger transmission coefficient $T$ for larger $V_{0}$ due to the occurrence of quantum interference effects. Thus, the result of the simulation reported in Fig. 16, supports the conclusion that doubling the amide I quanta 

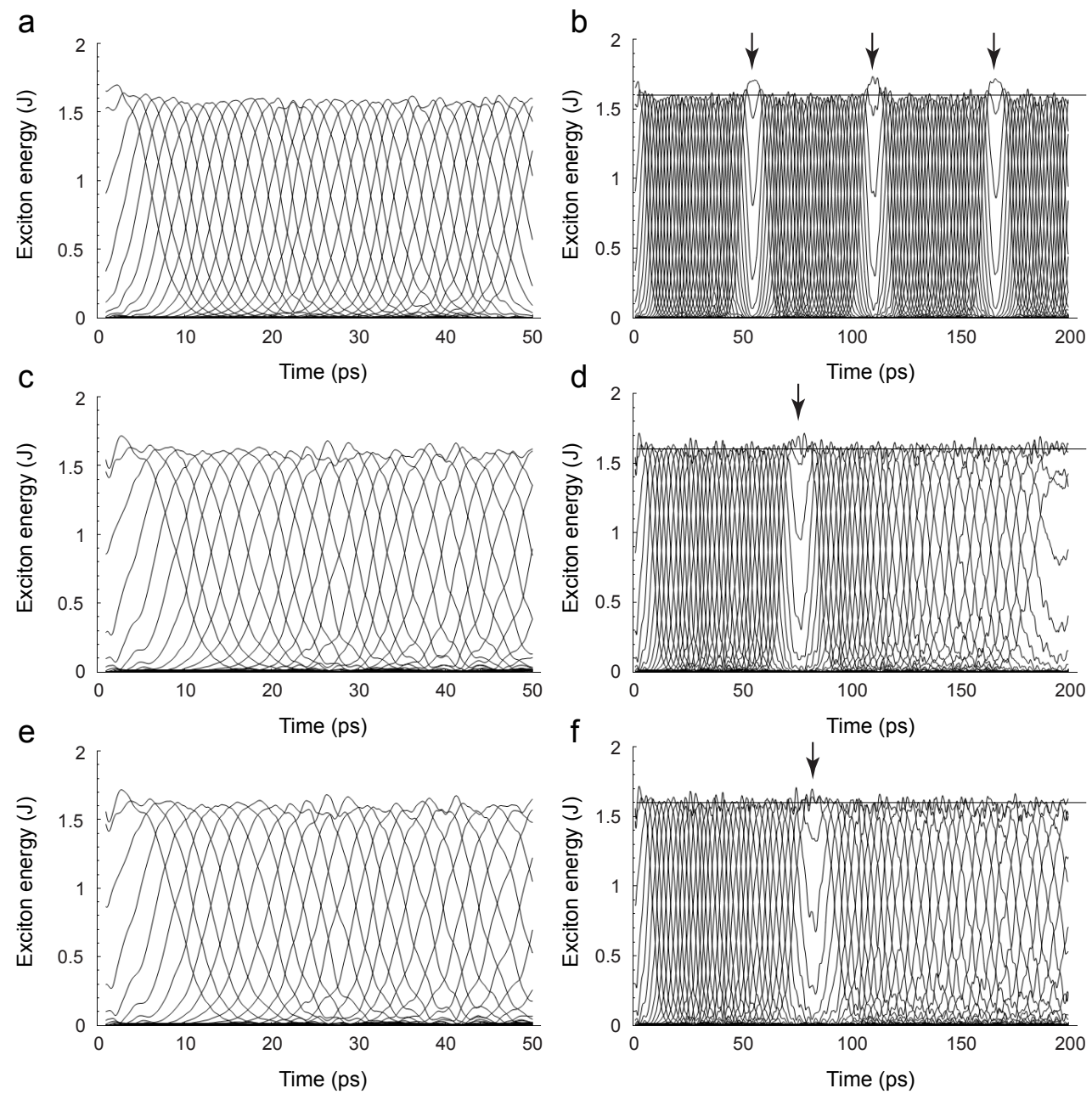

Figure 15: The quantum dynamics of the local exciton energy (measured in units of $J=0.155 \mathrm{zJ})$ computed for stretches of 5 terms $\mathcal{E}_{i}(t)$ in the sum for $\left|\left\langle\Psi\left|\hat{H}_{\text {ex }}\right| \Psi\right\rangle\right|$ of a Davydov soliton with $Q=1$ quantum of amide I energy for different values of exciton-phonon interaction isotropy $\xi$. (a-b) Exciton energy for $\xi=1$ soliton launched from the N-end of the $\alpha$-helix. (c-d) Exciton energy for $\xi=0$ soliton launched from the N-end of the $\alpha$-helix. (e-f) Exciton energy for $\xi=0$ soliton launched from the C-end of the $\alpha$-helix. The horizontal trend lines for $\mathcal{E}_{i}(t)$ peaks at $1.6 J$ show that the changes in soliton velocity are not accompanied by spread in the envelope of exciton quantum probability amplitudes. Arrows indicate soliton reflection from the $\alpha$-helix ends.

in the double soliton with $Q=2$, is analogous to raising the energy $E_{0}$ of massive particle that now faces a potential barrier with height $V_{0}<E_{0}$.

Because a particular effect of decreasing the isotropy of exciton-phonon interaction by lowering $\xi$, is to pin down the soliton, for the double soliton with $Q=2$, we were unable to test quantum tunneling through the massive barrier for $\xi<0.7$. In the case of $\xi=0.7$, the double soliton reflected from $200 M$ barrier (Fig. 17b) or $400 M$ barrier (Fig. 17k), yet it tunneled through the much more massive $600 M$ barrier (Fig. 17d). This behavior was qualitatively similar to the completely isotropic case with $\xi=1$. However, in comparison with the case $\xi=1$, for which the tunneling time was $71.1 \mathrm{ps}$ (Fig. 16d), the presence of some anisotropy for $\xi=0.7$ delayed the passage through the barrier with a tunneling time of 92.9 ps (Fig. $17 \mathrm{~d}$ ). Thus, the faster tunneling time for higher $\xi$ in double solitons with $Q=2$ 

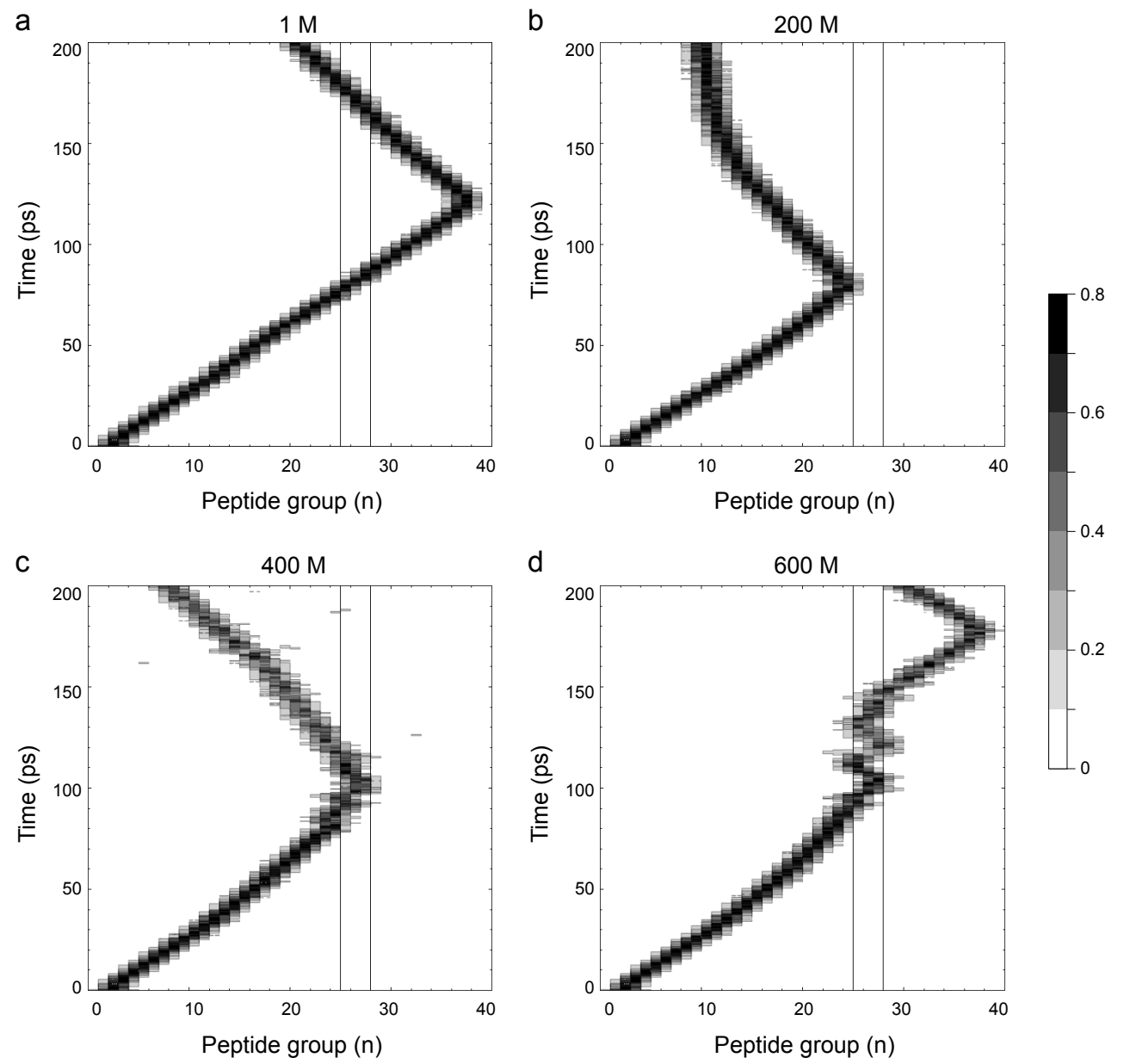

Figure 16: The quantum dynamics of a Davydov soliton with $Q=2$ quanta of amide I energy tunneling through or reflecting from a massive barrier located over three peptide groups $n=26-28$ visualized through the expectation value of the exciton number operator $Q\left|a_{n}\right|^{2}$ for $\xi=1$. Increasing the mass of the barrier may increase the interaction time with the barrier and increase the probability of tunneling through the barrier: no barrier $1 M$ (a), barrier in which each of the three peptide groups is with effective mass of $200 M$ (b), $400 M$ (c) and $600 M(\mathrm{~d})$. The barrier location is indicated with thin vertical lines.

differs from the observed tunneling times for single solitons with $Q=1$, and highlights the non-classical nature of soliton transmission through a potential barrier with $V_{0}<E_{0}$ due to manifested quantum interference effects in the transmission coefficient 40.

\subsection{Disorder effects on soliton stability}

The creation of soliton solutions by the system of Davydov equations (33) and (34) is made possible by the highly ordered protein $\alpha$-helix structure, which is reflected in the construction of the Hamiltonian (1). Because both living and non-living quantum physical systems are subject to the same fundamental quantum physical laws, we aimed at elucidating the importance of biological order 25,97,98 for outlining the quantum boundaries of life.

In most quantum chemistry applications, the Born-Oppenheimer approximation [99] allows the Schrödinger equation for a biomolecule to be separated it into two equations: i) an electronic Schrödinger equation, and ii) a nuclear Schrödinger equation. Only the positions 

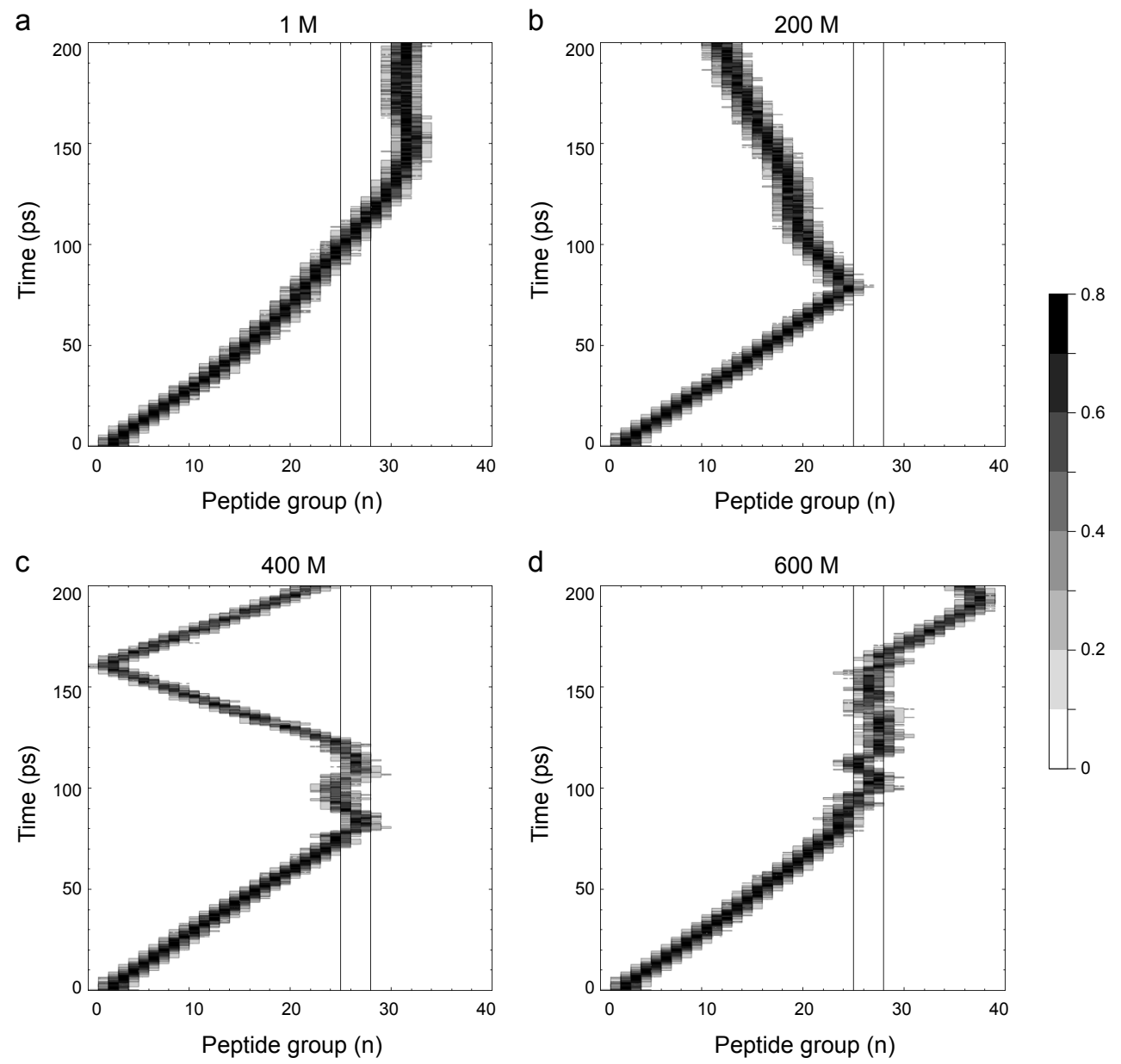

Figure 17: The quantum dynamics of a Davydov soliton with $Q=2$ quanta of amide I energy tunneling through or reflecting from a massive barrier located over three peptide groups $n=26-28$ visualized through the expectation value of the exciton number operator $Q\left|a_{n}\right|^{2}$ for $\xi=0.7$. Increasing the mass of the barrier may increase the interaction time with the barrier and increase the probability of tunneling through the barrier: no barrier $1 M$ (a), barrier in which each of the three peptide groups is with effective mass of $200 M$ (b), $400 M$ (c) and $600 M(\mathrm{~d})$. The barrier location is indicated with thin vertical lines.

of the much heavier atomic nuclei (not their momenta) generate the potential that enters in the Hamiltonian for solving the Schrödinger equation for the electrons [100]. Once the solution $\psi_{e}$ of the electronic Schrödinger equation is found, it is used to provide the potential energy function for the nuclear motion. The obtained vibrational nuclear wavefunctions $\psi_{n}$ with corresponding energies solve the nuclear Schrödinger equation. The total wavefunction $\Psi=\psi_{e} \psi_{n}$ for the biomolecule is then composed as a product of the electronic wavefunction $\psi_{e}$ and the nuclear wave function $\psi_{n}$. Further simplification of quantum mechanical calculations could be achieved with the Crude Born-Oppenheimer Approximation where the equilibrium separation of the nuclei is employed at all times.

The upshot of the Crude Born-Oppenheimer approximation manifests in the original Davydov Hamiltonian (1) in the guise of uniformity of dipole-dipole coupling energies $J_{n}$ between neighboring amide I oscillators, which are all set to $J=0.155 \mathrm{zJ}$ [56 59]. This introduces a high degree of stability in the biological system. In order to study the effects of instability on Davydov solitons, we have drawn randomly the value of each $J_{n}$ from a Gaussian 

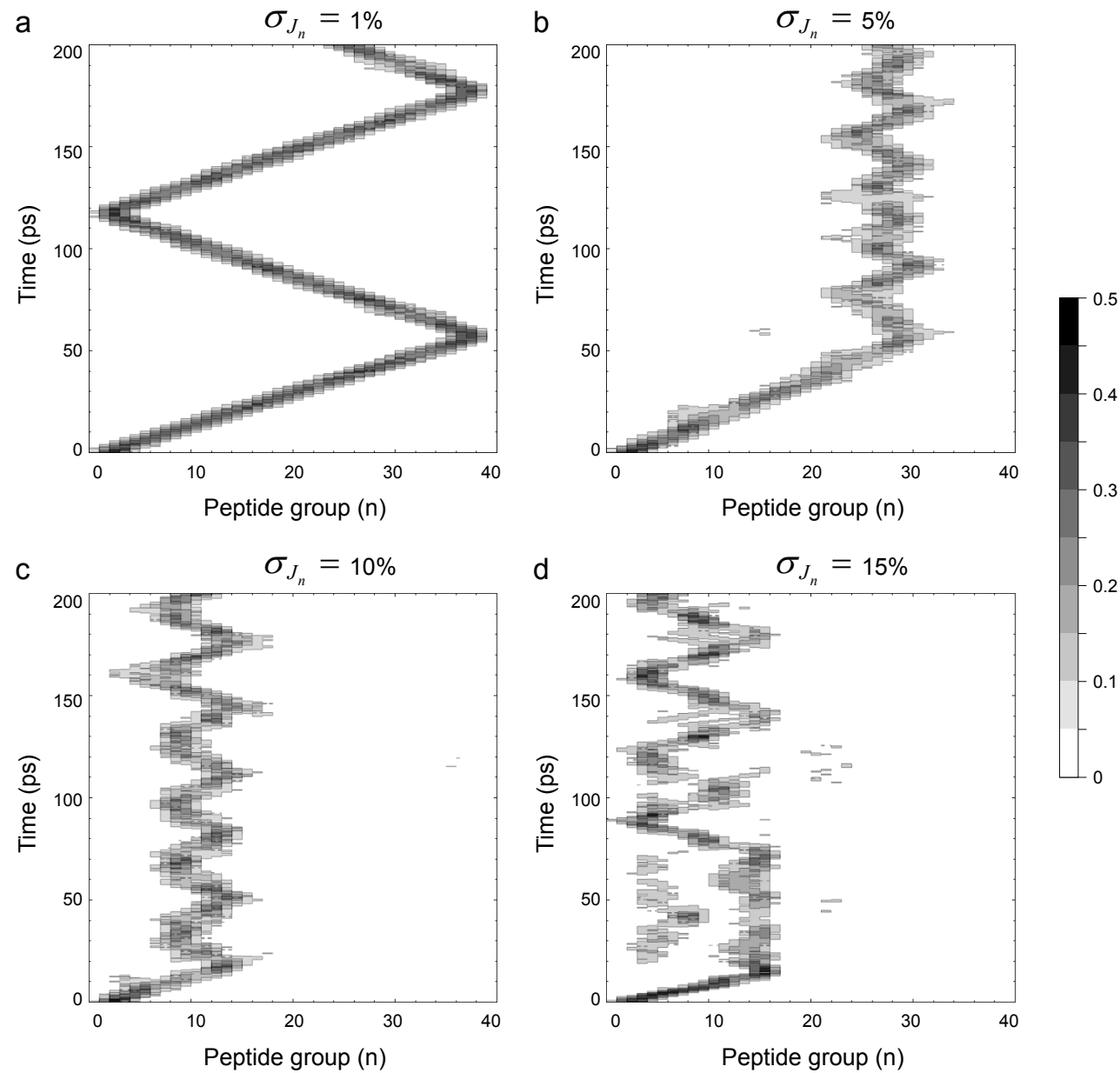

Figure 18: The quantum dynamics of a Davydov soliton with $Q=1$ quantum of amide I energy for protein $\alpha$-helix with randomly variable $J_{n}$ dipole-dipole coupling energy between amide I oscillators visualized through the expectation value of the exciton number operator $Q\left|a_{n}\right|^{2}$ for $\xi=1$. Non-uniformity of $J_{n}$ was quantified by the standard deviation $\sigma_{J_{n}}$ expressed as percentage from the mean value $J: \sigma_{J_{n}}=1 \%$ (a), $\sigma_{J_{n}}=5 \%$ (b), $\sigma_{J_{n}}=10 \%$ (c) and $\sigma_{J_{n}}=15 \%$ (d). Increasing the non-uniformity of $J_{n}$ destabilizes the soliton and traps it to a region flanked by low $J_{n}$ values.

distribution with mean value $J$, and standard deviation $\sigma_{J_{n}}$, defined as a percentage of $J$. To avoid reporting an outlier quantum dynamics, we have simulated at least 5 randomly drawn distributions for a given $\sigma_{J_{n}}$ and presented the outcome of a simulation run, which had been supported by another visually similar outcome.

For a protein $\alpha$-helix with completely isotropic exciton-phonon interaction $\xi=1$, the presence of a dipole-dipole coupling disorder $\sigma_{J_{n}}<5 \%$ was not detrimental for the moving soliton (Fig. 18 a). When the disorder was increased between $\sigma_{J_{n}}=5 \%$ (Fig. 18p) and $\sigma_{J_{n}}=$ $10 \%$ (Fig. 18k), the soliton propagated along the protein $\alpha$-helix until it was trapped inside a region flanked by low $J_{n}$ values. Visually, the quantum dynamics of the soliton resembled compartmentalization inside a short protein $\alpha$-helix rather than pinning. For greater disorder $\sigma_{J_{n}}=15 \%$, features of soliton disintegration were observed even though part of the soliton still persisted (Fig. 18d).

For a protein $\alpha$-helix with completely anisotropic exciton-phonon interaction $\xi=0$, the presence of dipole-dipole coupling disorder had similar effects, however the soliton appeared 

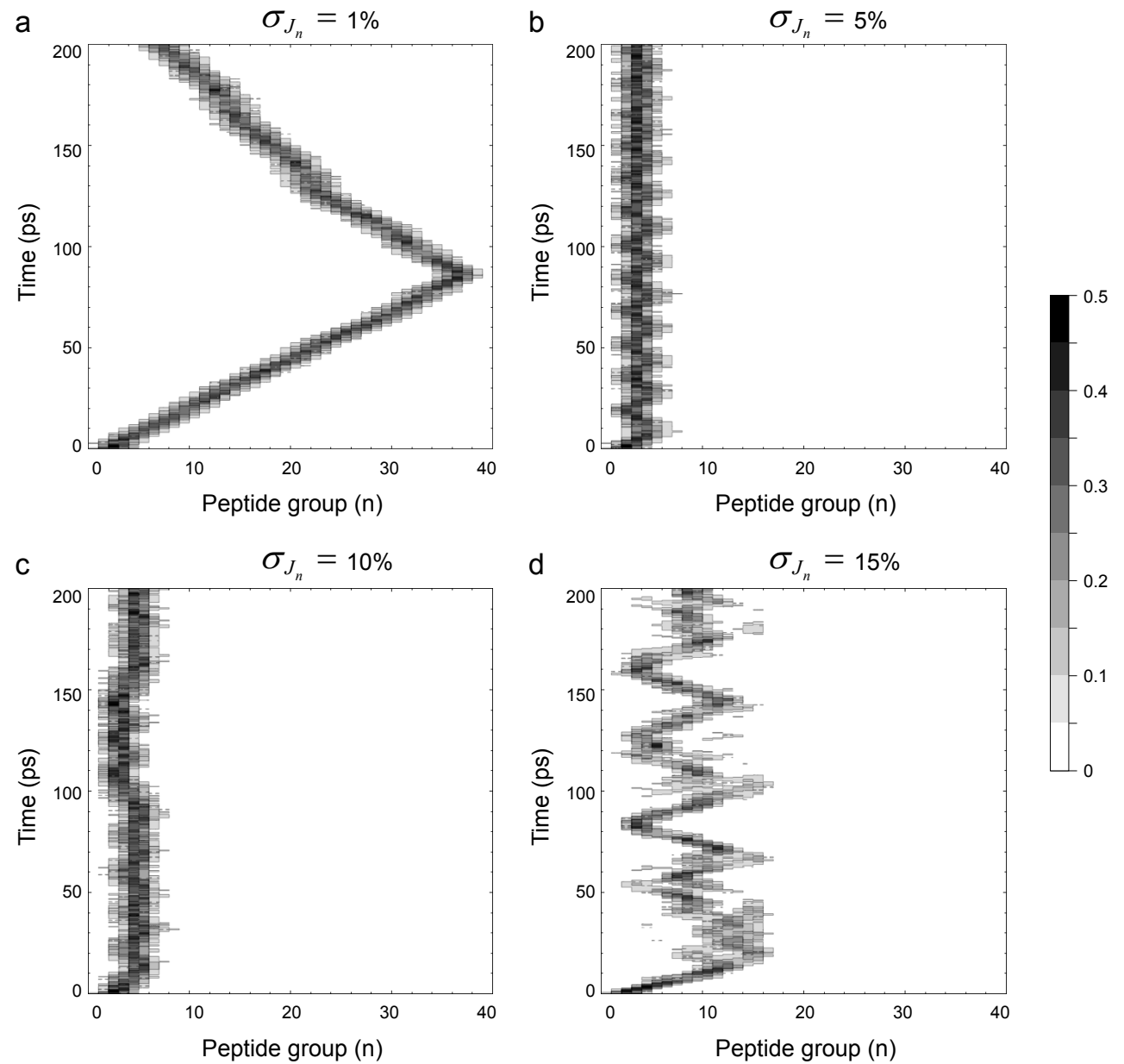

Figure 19: The quantum dynamics of a Davydov soliton with $Q=1$ quantum of amide I energy for protein $\alpha$-helix with randomly variable $J_{n}$ dipole-dipole coupling energy between amide I oscillators visualized through the expectation value of the exciton number operator $Q\left|a_{n}\right|^{2}$ for $\xi=0$. Non-uniformity of $J_{n}$ was quantified by the standard deviation $\sigma_{J_{n}}$ expressed as percentage from the mean value $J: \sigma_{J_{n}}=1 \%$ (a), $\sigma_{J_{n}}=5 \%$ (b), $\sigma_{J_{n}}=10 \%$ (c) and $\sigma_{J_{n}}=15 \%$ (d). Increasing the non-uniformity of $J_{n}$ destabilizes the soliton.

to be pinned for $\sigma_{J_{n}}=5 \%$ (Fig. $19 \mathrm{~b}$ ) and $\sigma_{J_{n}}=10 \%$ (Fig. 19.). The destabilization of the soliton at $\sigma_{J_{n}}=15 \%$ was present (Fig. $19 \mathrm{~d}$ ), albeit a bit weaker compared with the completely isotropic $\xi=1$ case. Thus, the order and uniformity of dipole-dipole coupling energies between neighboring amide I oscillators is pivotal for the highly efficient, dissipationless, solitonic transport of energy by protein $\alpha$-helices. In addition, cooperative effects between backbone dipole-dipole interactions are instrumental for the formation of secondary and supersecondary structures of proteins, and after successful folding keep proteins in their folded structural state [101, while noting that protein conformational transitions, as microscopic biochemical processes, a fortiori, involve quantum states (see e.g. 102]).

To illustrate the fact that not all types of disorder are equally adverse to soliton dynamics, we have also randomly varied the amino acid masses $M_{n}$ within the maximal biochemical range of $\sigma_{M_{n}}=25 \%$ in a protein $\alpha$-helix. For all values of $\xi$, the solitons readily propagated along the $\alpha$-helix spine (Fig. 20) and were virtually indistinguishable from the case with uniform amino acid masses (cf. Fig. 12 a vs Fig. 20a and Fig. 13 a vs Fig. 20d). 

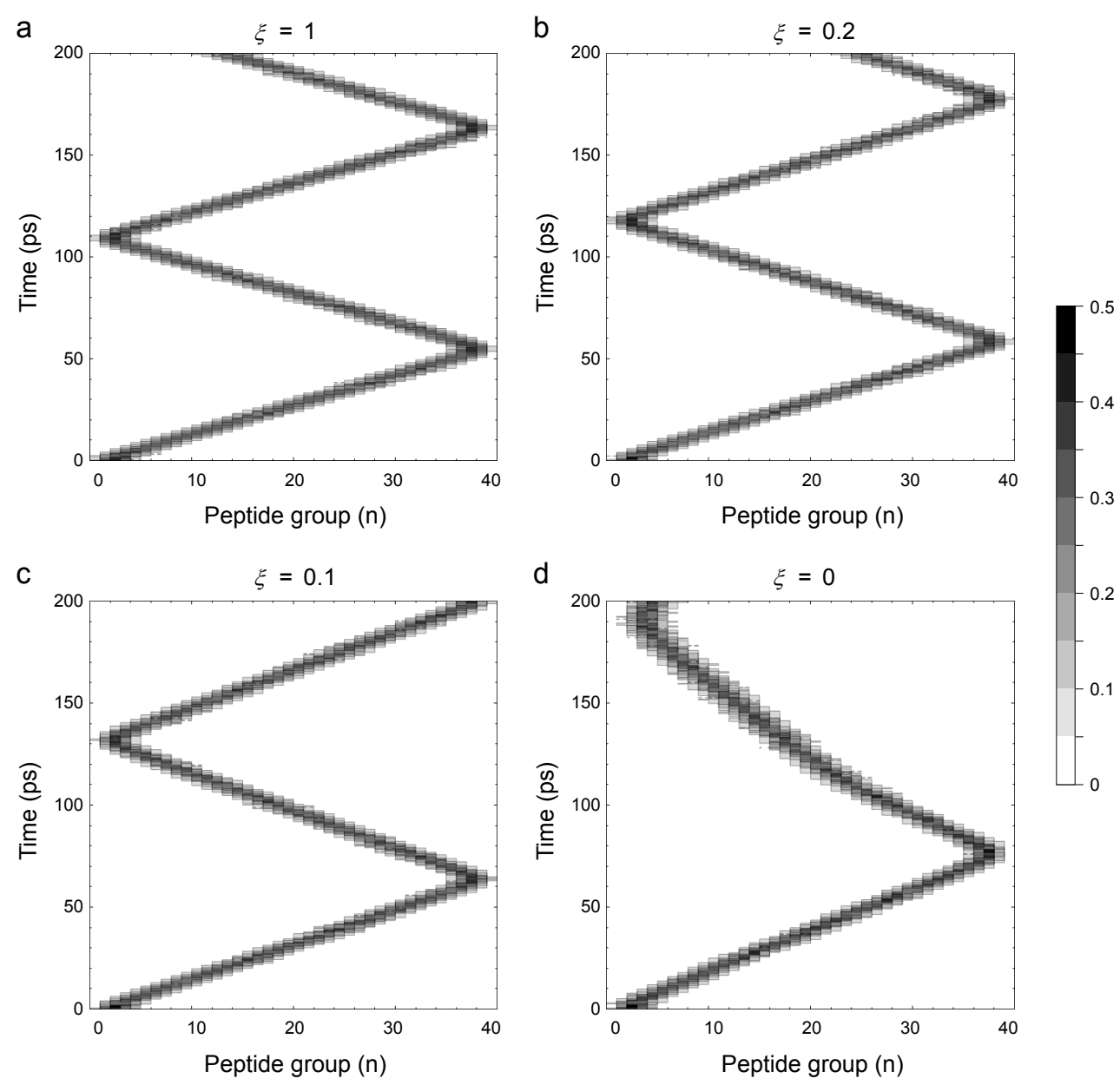

Figure 20: The quantum dynamics of a Davydov soliton with $Q=1$ quantum of amide I energy for protein $\alpha$-helix with nonuniform $M_{n}$ peptide group masses (standard deviation $\sigma_{M_{n}}$ is $25 \%$ from the mean value $M=1.9 \times 10^{-25} \mathrm{~kg}$ ) visualized through the expectation value of the exciton number operator $Q\left|a_{n}\right|^{2}$ for different values of the isotropy of excitonphonon interaction: $\xi=1$ (a), $\xi=0.2(\mathrm{~b}), \xi=0.1$ (c) and $\xi=0$ (d).

\section{Conclusions}

The importance of proteins for maintaining life cannot be overstated [103,104]. The majority of biological processes are catalyzed or performed by proteins, and the main part of the coding DNA in the genome is dedicated to storing hereditary information for the production of proteins. The underlying mechanisms behind the versatility of protein function, however, remained elusive within the deterministic clockwork structures of classical physics [25, 97]. In an attempt to address the perceived theoretical crisis in bioenergetics, Alexander Davydov, in 1973, proposed that the amide I quanta of peptide vibrational energy $(\mathrm{C}=\mathrm{O}$ stretching $)$ might become self-localized through interactions with lattice phonons in protein $\alpha$-helices [55]. The original Davydov model was analytically studied with the use of the continuum approximation or simulated numerically [56 68], and then further generalized to include explicit treatment of multiple amide I quanta and the possible anisotropies of various physical parameters in realistic protein models $54,69,80,86,91,92$.

To gain insights into the transport and utilization of energy by proteins, in this work we have studied the quantum dynamics of multiple amide I quanta, which arises from solving 
the Schrödinger equation for the generalized Davydov Hamiltonian (1). Utilizing only standard quantum mechanical commutators and the Schrödinger equation, we initially derived a discrete system of generalized Davydov equations (33) and (34) that govern the quantum dynamics of amide I excitons in protein $\alpha$-helices (Section 3). Then, we performed computational simulations, which have revealed that the discrete system of Davydov equations supports the corresponding solitons, even for ultrashort protein $\alpha$-helices whose length is only 10 peptide groups (Section 4.2). This suggests that natural evolution is able to select optimal protein $\alpha$-helix lengths in gradual steps without losing the soliton mechanism. Next, we have found that soliton collisions could lead to either persistent pinned solitons, or to generating intermittent peaks of concentrated amide I energy through constructive quantum interference (Section 4.3). This provides a quantum physical mechanism for delivery of concentrated peaks of energy at protein active centers. The free energy delivered by the soliton to the active protein center may then be utilized to do physical work triggering chemical reactions and classical processes on nano-, micro- or milli-second timescales. The physical mechanisms for amplification of individual quantum processes to trigger macroscopic classical events at these slower timescales, which will effectively constitute the quantum-to-classical transition, were not covered in the current study and deserve further theoretical modeling along the lines of macromolecular electron clouds interacting with each other or with the electromagnetic field in biological systems $68,105,106$. Elaborate macro-quantum modeling of multiple proteins and their interaction with the quantized electromagnetic field would be particularly relevant for the function of protein voltage-gated ion channels incorporated in neuronal plasma membranes, where the electric field may reach values on the order of $10^{7} \mathrm{~V} / \mathrm{m}$ during the course of action potentials fired by the neurons 21, 107.

In addition to participation in quantum interference phenomena, the quantum nature of Davydov solitons was also illustrated by their capability to tunnel through massive barriers applied by external protein clamps (Section 4.4). This allows proteins to accomplish otherwise classically impossible tasks. Lastly, we have demonstrated the importance of the biological order for soliton stability through the regular helical geometry that ensures uniform dipoledipole coupling energies between neighboring amide I oscillators (Section 4.5). Interestingly, the presence of disorder in the dipole-dipole coupling energies did not cause direct soliton disintegration, but instead resulted in compartmentalization of the solitons within protein segments flanked with low dipole-dipole couplings. This may have been valuable for the natural evolution of protein function because random coil domains could evolve into $\alpha$-helices, or vice versa, thereby sculpturing-out either energy transmission lines or protein active centers for utilization of the delivered energy.

The present findings may also shed new light on the abiotic origin of life where randomly assembled polypeptides could have found a way to preserve existing seeds of order in the first replicators. Of course, the origin of life is the most challenging problem of all time [108] and we may never find out how exactly life started as a consequence of the very nature of the evolutionary process - we can only see the best survivors having lost forever all those less fit ancestors that were replaced in natural history. From living organisms that can fossilize, we have been lucky to have a glimpse at what life was in past eras. To look further back to the dawn of precellular life with the first biomolecular replicators, however, is an uncertain task since single biomolecules leave no traces, and the conditions on the early surface of the planet (Earth) are likely to have been quite different from those which we currently imagine [109]. There is evidence, however, that a network of cross-replicating molecules is more robust, and operates faster than a single self-replicator [110,111]. Regardless of whether polypeptides were present from the outset of life [112], or at a later stage joined up with the biomolecular repertoire of living systems, our results on the quantum dynamics of Davydov 
solitons in protein $\alpha$-helices provide evidence for the non-trivial role of quantum effects in maintaining life, and therefore highlight the prominent role of biological order to foster those quantum effects. These events also contend that the first replicators should have had lines for highly efficient quantum transmission of energy, capable of trapping energy quanta in active centers, and the prowess to utilize seeds of order to sustain non-trivial quantum dynamics at biochemically relevant timescales.

\section{References}

[1] A. I. Oparin. The Origin of Life on the Earth. Academic Press, New York, 1957.

[2] D. E. Koshland. The seven pillars of life. Science 2002; 295(5563):2215-2216. doi: $10.1126 /$ science. 1068489

[3] E. N. Trifonov. Vocabulary of definitions of life suggests a definition. Journal of Biomolecular Structure and Dynamics 2011; 29(2):259-266. doi:10.1080/ 073911011010524992 .

[4] B. Kuhlman, P. Bradley. Advances in protein structure prediction and design. Nature Reviews Molecular Cell Biology 2019; 20(11):681-697. doi:10.1038/ s41580-019-0163-x.

[5] J. F. Atkins, R. Gesteland. The 22nd amino acid. Science 2002; 296(5572):1409-1410. doi:10.1126/science.1073339.

[6] L. Pauling, R. B. Corey, H. R. Branson. The structure of proteins: two hydrogen-bonded helical configurations of the polypeptide chain. Proceedings of the National Academy of Sciences 1951; 37(4):205-211. doi:10.1073/pnas.37.4.205.

[7] L. Pauling, R. B. Corey. The pleated sheet, a new layer configuration of polypeptide chains. Proceedings of the National Academy of Sciences 1951; 37(5):251-256. doi: 10.1073/pnas.37.5.251.

[8] R. S. Vieira-Pires, J. H. Morais-Cabral. $3_{10}$ helices in channels and other membrane proteins. Journal of General Physiology 2010; 136(6):585-592. doi:10.1085/jgp. 201010508

[9] P. Kumar, M. Bansal. Dissecting $\pi$-helices: sequence, structure and function. The FEBS Journal 2015; 282(22):4415-4432. doi:10.1111/febs.13507.

[10] E. Guseva, R. N. Zuckermann, K. A. Dill. Foldamer hypothesis for the growth and sequence differentiation of prebiotic polymers. Proceedings of the National Academy of Sciences 2017; 114(36):E7460-E7468. doi:10.1073/pnas.1620179114.

[11] J. W. Schopf, K. Kitajima, M. J. Spicuzza, A. B. Kudryavtsev, J. W. Valley. SIMS analyses of the oldest known assemblage of microfossils document their taxon-correlated carbon isotope compositions. Proceedings of the National Academy of Sciences 2018; 115(1):53-58. doi:10.1073/pnas.1718063115.

[12] M. J. Schnitzer, S. M. Block. Kinesin hydrolyses one ATP per 8-nm step. Nature 1997; 388(6640):386-390. doi:10.1038/41111. 
[13] D. L. Coy, M. Wagenbach, J. Howard. Kinesin takes one 8-nm step for each ATP that it hydrolyzes. Journal of Biological Chemistry 1999; 274(6):3667-3671. doi:10.1074/ jbc.274.6.3667.

[14] M. Ahn, D. Beacham, R. E. Westenbroek, T. Scheuer, W. A. Catterall. Regulation of $\mathrm{Na}_{V} 1.2$ channels by brain-derived neurotrophic factor, TrkB, and associated Fyn kinase. Journal of Neuroscience 2007; 27(43):11533-11542. doi:10.1523/jneurosci. 5005-06.2007.

[15] K. Tucker, D. A. Fadool. Neurotrophin modulation of voltage-gated potassium channels in rat through TrkB receptors is time and sensory experience dependent. Journal of Physiology 2002; 542(2):413-429. doi:10.1113/jphysiol.2002.017376.

[16] A. Szöke, J. Hajdu. Energy utilization in fluctuating biological energy converters. Structural Dynamics 2016; 3(3):034701. doi:10.1063/1.4945792.

[17] K. Matsuno. Forming and maintaining a heat engine for quantum biology. Biosystems 2006; 85(1):23-29. doi:10.1016/j.biosystems.2006.02.002.

[18] K. Matsuno. From quantum measurement to biology via retrocausality. Progress in Biophysics and Molecular Biology 2017; 131:131-140. doi:10.1016/j.pbiomolbio. 2017.06.012.

[19] A. V. Melkikh, A. Khrennikov. Nontrivial quantum and quantum-like effects in biosystems: unsolved questions and paradoxes. Progress in Biophysics and Molecular Biology 2015; 119(2):137-161. doi:10.1016/j.pbiomolbio.2015.07.001.

[20] A. Marais, B. Adams, A. K. Ringsmuth, M. Ferretti, J. M. Gruber, R. Hendrikx, M. Schuld, S. L. Smith, I. Sinayskiy, T. P. J. Krüger, F. Petruccione, R. van Grondelle. The future of quantum biology. Journal of The Royal Society Interface 2018; 15(148). doi:10.1098/rsif.2018.0640.

[21] D. D. Georgiev. Quantum Information and Consciousness: A Gentle Introduction. CRC Press, Boca Raton, 2017. doi:10.1201/9780203732519.

[22] D. D. Georgiev. Inner privacy of conscious experiences and quantum information. Biosystems 2020; 187:104051. doi:10.1016/j.biosystems.2019.104051.

[23] D. D. Georgiev, J. F. Glazebrook. Subneuronal processing of information by solitary waves and stochastic processes. in: S. E. Lyshevski (Ed.), Nano and Molecular Electronics Handbook. CRC Press, Boca Raton, 2007. Ch. 17. pp. 1-41.

[24] D. D. Georgiev, J. F. Glazebrook. Quasiparticle tunneling in neurotransmitter release. in: W. A. Goddard III, D. Brenner, S. E. Lyshevski, G. J. Iafrate (Eds.), Handbook of Nanoscience, Engineering, and Technology. 3rd Edition. CRC Press, Boca Raton, 2012. Ch. 30. pp. 983-1016.

[25] E. Schrödinger. What is Life? The Physical Aspect of the Living Cell \& Mind and Matter. Cambridge University Press, Cambridge, 1977.

[26] N. Lambert, Y.-N. Chen, Y.-C. Cheng, C.-M. Li, G.-Y. Chen, F. Nori. Quantum biology. Nature Physics 2013; 9(1):10-18. doi:10.1038/nphys2474. 
[27] J. C. Brookes. Quantum effects in biology: golden rule in enzymes, olfaction, photosynthesis and magnetodetection. Proceedings of the Royal Society A 2017; 473:20160822. doi:10.1098/rspa.2016.0822.

[28] D. De Vault. Quantum-Mechanical Tunneling in Biological Systems. Vol. 2. Cambridge University Press, Cambridge, UK, 1984.

[29] M. J. Sutcliffe, N. S. Scrutton. Enzyme catalysis: over-the-barrier or throughthe-barrier?. Trends in Biochemical Sciences 2000; 25(9):405-408. doi:10.1016/ S0968-0004(00) 01642-X.

[30] J. Basran, S. Patel, M. J. Sutcliffe, N. S. Scrutton. Importance of barrier shape in enzyme-catalyzed reactions: vibrationally assisted hydrogen tunneling in tryptophan tryptophylquinone-dependent amine dehydrogenases. Journal of Biological Chemistry 2001; 276(9):6234-6242. doi:10.1074/jbc.M008141200.

[31] G. R. Fleming, G. D. Scholes, Y.-C. Cheng. Quantum effects in biology. Procedia Chemistry 2011; 3:38-57.

[32] S. Ichinose. Soliton excitations in alpha-helical protein structures. Chaos, Solitons 86 Fractals 1991; 1(6):501-509. doi:10.1016/0960-0779(91)90040-G.

[33] A. S. Edison. Linus Pauling and the planar peptide bond. Nature Structural Biology 2001; 8(3):201-202. doi:10.1038/84921.

[34] J. Kovac. A weird insult from Norway: Linus Pauling as public intellectual. Soundings: An Interdisciplinary Journal 1999; 82(1-2):91-106.

[35] A. Barth. Infrared spectroscopy of proteins. Biochimica et Biophysica Acta (BBA) Bioenergetics 2007; 1767(9):1073-1101. doi:10.1016/j.bbabio.2007.06.004.

[36] D. S. Sholl, J. A. Steckel. Density Functional Theory: A Practical Introduction. John Wiley \& Sons, Hoboken, New Jersey, 2009.

[37] S. K. Kolev, P. S. Petkov, M. Rangelov, G. N. Vayssilov. Ab initio molecular dynamics of $\mathrm{Na}^{+}$and $\mathrm{Mg}^{2+}$ countercations at the backbone of RNA in water solution. ACS Chemical Biology 2013; 8(7):1576-1589. doi:10.1021/cb300463h.

[38] S. K. Kolev, P. S. Petkov, M. A. Rangelov, D. V. Trifonov, T. I. Milenov, G. N. Vayssilov. Interaction of $\mathrm{Na}^{+}, \mathrm{K}^{+}, \mathrm{Mg}^{2+}$ and $\mathrm{Ca}^{2+}$ counter cations with RNA. Metallomics 2018; 10(5):659-678. doi:10.1039/c8mt00043c

[39] C. N. Pace, H. Fu, K. Lee Fryar, J. Landua, S. R. Trevino, D. Schell, R. L. Thurlkill, S. Imura, J. M. Scholtz, K. Gajiwala, J. Sevcik, L. Urbanikova, J. K. Myers, K. Takano, E. J. Hebert, B. A. Shirley, G. R. Grimsley. Contribution of hydrogen bonds to protein stability. Protein Science 2014; 23(5):652-661. doi:10.1002/pro.2449.

[40] N. S. Myshakina, Z. Ahmed, S. A. Asher. Dependence of amide vibrations on hydrogen bonding. Journal of Physical Chemistry B 2008; 112(38):11873-11877. doi:10.1021/ jp8057355

[41] X.-Z. Li, B. Walker, A. Michaelides. Quantum nature of the hydrogen bond. Proceedings of the National Academy of Sciences 2011; 108(16):6369-6373. doi:10.1073/pnas. 1016653108 
[42] O. Pusuluk, T. Farrow, C. Deliduman, K. Burnett, V. Vedral. Proton tunnelling in hydrogen bonds and its implications in an induced-fit model of enzyme catalysis. Proceedings of the Royal Society A 2018; 474(2218):20180037. doi:10.1098/rspa.2018.0037.

[43] O. Pusuluk, G. Torun, C. Deliduman. Quantum entangelemnt shared in hydrogen bonds and its usage as a resource in molecular recognition. Modern Physics Letters B 2018; 32(26):1850308. doi:10.1142/S0217984918503086.

[44] S. Krimm, J. Bandekar. Vibrational spectroscopy and conformation of peptides, polypeptides, and proteins. Advances in Protein Chemistry 1986; 38:181-364. doi: 10.1016/S0065-3233(08)60528-8.

[45] P. Hamm, M. Lim, R. M. Hochstrasser. Structure of the amide I band of peptides measured by femtosecond nonlinear-infrared spectroscopy. Journal of Physical Chemistry B 1998; 102(31):6123-6138. doi:10.1021/jp9813286.

[46] E. S. Manas, Z. Getahun, W. W. Wright, W. F. DeGrado, J. M. Vanderkooi. Infrared spectra of amide groups in $\alpha$-helical proteins: evidence for hydrogen bonding between helices and water. Journal of the American Chemical Society 2000; 122(41):9883-9890. doi:10.1021/ja001782z.

[47] P. M. Wiggins. Role of water in some biological processes. Microbiological Reviews 1990; 54(4):432-449.

[48] P. M. Wiggins. Life depends on two kinds of water. PloSOne 2008; 3(1):e1406. doi: 10.1371/journal.pone.0001406.

[49] H. Yang, S. Yang, J. Kong, A. Dong, S. Yu. Obtaining information about protein secondary structures in aqueous solution using Fourier transform IR spectroscopy. Nature Protocols 2015; 10(3):382-396. doi:10.1038/nprot.2015.024.

[50] J. Edler, P. Hamm, A. C. Scott. Femtosecond study of self-trapped vibrational excitons in crystalline acetanilide. Physical Review Letters 2002; 88(6):067403. doi:10.1103/ PhysRevLett.88.067403.

[51] J. Edler, P. Hamm. Self-trapping of the amide I band in a peptide model crystal. Journal of Chemical Physics 2002; 117(5):2415-2424. doi:10.1063/1.1487376.

[52] J. Edler, R. Pfister, V. Pouthier, C. Falvo, P. Hamm. Direct observation of self-trapped vibrational states in $\alpha$-helices. Physical Review Letters 2004; 93(10):106405. doi:10. 1103/PhysRevLett.93.106405.

[53] J. Edler, P. Hamm. Spectral response of crystalline acetanilide and $N$-methylacetamide: vibrational self-trapping in hydrogen-bonded crystals. Physical Review B 2004; 69(21):214301. doi:10.1103/PhysRevB.69.214301.

[54] D. D. Georgiev, J. F. Glazebrook. On the quantum dynamics of Davydov solitons in protein $\alpha$-helices. Physica A: Statistical Mechanics and its Applications 2019; 517:257269. doi:10.1016/j.physa.2018.11.026.

[55] A. S. Davydov. The theory of contraction of proteins under their excitation. Journal of Theoretical Biology 1973; 38(3):559-569. doi:10.1016/0022-5193(73)90256-7.

[56] A. S. Davydov, N. I. Kislukha. Solitons in one-dimensional molecular chains. Physica Status Solidi (b) 1976; 75(2):735-742. doi:10.1002/pssb.2220750238. 
[57] A. S. Davydov. Solitons in molecular systems. Physica Scripta 1979; 20(3-4):387-394. doi:10.1088/0031-8949/20/3-4/013.

[58] A. S. Davydov. Solitons in quasi-one-dimensional molecular structures. Soviet Physics Uspekhi 1982; 25(12):898-918. doi:10.1070/pu1982v025n12abeh005012.

[59] A. S. Davydov. Quantum theory of the motion of a quasi-particle in a molecular chain with thermal vibrations taken into account. Physica Status Solidi (b) 1986; 138(2):559576. doi:10.1002/pssb.2221380221.

[60] A. S. Davydov, V. N. Ermakov. Linear and nonlinear resonance electron tunneling through a system of potential barriers. Physica D: Nonlinear Phenomena 1987; 28(1):168-180. doi:10.1016/0167-2789(87)90127-8.

[61] A. S. Davydov, V. N. Ermakov. Soliton generation at the boundary of a molecular chain. Physica D: Nonlinear Phenomena 1988; 32(2):318-323. doi:10.1016/0167-2789(88) 90059-0.

[62] L. S. Brizhik, A. S. Davydov. Soliton excitations in one-dimensional molecular systems. Physica Status Solidi (b) 1983; 115(2):615-630. doi:10.1002/pssb.2221150233.

[63] L. S. Brizhik, Y. B. Gaididei, A. A. Vakhnenko, V. A. Vakhnenko. Soliton generation in semi-infinite molecular chains. Physica Status Solidi (b) 1988; 146(2):605-612. doi: $10.1002 / \mathrm{pssb} .2221460221$.

[64] L. S. Brizhik. Soliton generation in molecular chains. Physical Review B 1993; 48(5):3142-3144. doi:10.1103/PhysRevB.48.3142.

[65] L. S. Brizhik, A. A. Eremko. Electron autolocalized states in molecular chains. Physica D: Nonlinear Phenomena 1995; 81(3):295-304. doi:10.1016/0167-2789(94)00206-6.

[66] L. S. Brizhik, A. Eremko, B. Piette, W. Zakrzewski. Solitons in $\alpha$-helical proteins. Physical Review E 2004; 70(3):031914. doi:10.1103/PhysRevE.70.031914.

[67] L. Brizhik, A. Eremko, B. Piette, W. Zakrzewski. Charge and energy transfer by solitons in low-dimensional nanosystems with helical structure. Chemical Physics 2006; 324(1):259-266. doi:10.1016/j. chemphys.2006.01.033.

[68] L. Brizhik, A. Eremko, B. Piette, W. Zakrzewski. Ratchet effect of Davydov's solitons in nonlinear low-dimensional nanosystems. International Journal of Quantum Chemistry $2010 ; 110(1): 25-37$. doi:10.1002/qua.22083.

[69] L. Cruzeiro, J. Halding, P. L. Christiansen, O. Skovgaard, A. C. Scott. Temperature effects on the Davydov soliton. Physical Review A 1988; 37(3):880-887. doi:10.1103/ PhysRevA.37.880.

[70] L. Cruzeiro-Hansson, V. M. Kenkre. Localized versus delocalized ground states of the semiclassical Holstein Hamiltonian. Physics Letters A 1994; 190(1):59-64. doi:10. 1016/0375-9601(94)90366-2.

[71] L. Cruzeiro-Hansson, S. Takeno. Davydov model: the quantum, mixed quantumclassical, and full classical systems. Physical Review E 1997; 56(1):894-906. doi: 10.1103/PhysRevE.56.894 
[72] L. Cruzeiro. The Davydov/Scott model for energy storage and transport in proteins. Journal of Biological Physics 2009; 35(1):43-55. doi:10.1007/s10867-009-9129-0.

[73] W. C. Kerr, P. S. Lomdahl. Quantum-mechanical derivation of the equations of motion for Davydov solitons. Physical Review B 1987; 35(7):3629-3632. doi:10.1103/ PhysRevB.35.3629.

[74] W. C. Kerr, P. S. Lomdahl. Quantum-mechanical derivation of the Davydov equations for multi-quanta states. in: P. L. Christiansen, A. C. Scott (Eds.), Davydov's Soliton Revisited: Self-Trapping of Vibrational Energy in Protein. Springer, New York, 1990. pp. 23-30. doi:10.1007/978-1-4757-9948-4_2.

[75] L. MacNeil, A. C. Scott. Launching a Davydov soliton: II. Numerical studies. Physica Scripta 1984; 29(3):284-287. doi:10.1088/0031-8949/29/3/017.

[76] A. C. Scott. Launching a Davydov soliton: I. Soliton analysis. Physica Scripta 1984; 29(3):279-283. doi:10.1088/0031-8949/29/3/016.

[77] A. C. Scott. Davydov solitons in polypeptides. Philosophical Transactions of the Royal Society of London Series A, Mathematical and Physical Sciences 1985; 315(1533):423436. doi:10.1098/rsta.1985.0049.

[78] A. C. Scott. Davydov's soliton. Physics Reports 1992; 217(1):1-67. doi:10.1016/ 0370-1573(92) 90093-F.

[79] B. Luo, J. Ye, Y. Zhao. Variational study of polaron dynamics with the Davydov ansätze. Physica Status Solidi (c) 2011; 8(1):70-73. doi:10.1002/pssc.201000721.

[80] J. Luo, B. Piette. A generalised Davydov-Scott model for polarons in linear peptide chains. European Physical Journal B 2017; 90(8):155. doi:10.1140/epjb/ e2017-80209-2.

[81] J. Sun, B. Luo, Y. Zhao. Dynamics of a one-dimensional Holstein polaron with the Davydov ansätze. Physical Review B 2010; 82(1):014305. doi:10.1103/PhysRevB.82. 014305 .

[82] D. Hennig. Energy transport in $\alpha$-helical protein models: one-strand versus three-strand systems. Physical Review B 2002; 65(17):174302. doi:10.1103/PhysRevB.65.174302.

[83] L. S. Brizhik, J. Luo, B. M. A. G. Piette, W. J. Zakrzewski. Long-range donor-acceptor electron transport mediated by $\alpha$-helices. Physical Review E 2019; 100(6):062205. doi: 10.1103/PhysRevE.100.062205.

[84] T. Holstein. Studies of polaron motion: Part I. The molecular-crystal model. Annals of Physics 1959; 8(3):325-342. doi:10.1016/0003-4916(59)90002-8.

[85] T. Holstein. Studies of polaron motion: Part II. The "small" polaron. Annals of Physics 1959; 8(3):343-389. doi:10.1016/0003-4916(59)90003-X.

[86] D. D. Georgiev, J. F. Glazebrook. Quantum tunneling of Davydov solitons through massive barriers. Chaos, Solitons and Fractals 2019; 123:275-293. doi:10.1016/j. chaos.2019.04.013.

[87] S. Tauber. On multinomial coefficients. The American Mathematical Monthly 1963; 70(10):1058-1063. doi:10.1080/00029890.1963.11992172. 
[88] P. A. M. Dirac. The Principles of Quantum Mechanics. 4th Edition. Oxford University Press, Oxford, 1967.

[89] D. D. Georgiev, J. F. Glazebrook. Neurotransmitter release and conformational changes within the SNARE protein complex. in: S. E. Lyshevski (Ed.), Nanoengineering, Quantum Science, and, Nanotechnology Handbook. CRC Press, Boca Raton, 2019. pp. 375404.

[90] Y. S. Kivshar, B. A. Malomed. Dynamics of solitons in nearly integrable systems. Reviews of Modern Physics 1989; 61(4):763-915. doi:10.1103/RevModPhys.61.763.

[91] W. Förner, J. Ladik. Influence of heat bath and disorder on Davydov solitons. in: P. L. Christiansen, A. C. Scott (Eds.), Davydov's Soliton Revisited: Self-Trapping of Vibrational Energy in Protein. Springer, New York, 1990. pp. 267-283. doi: 10.1007/978-1-4757-9948-4_20.

[92] W. Förner. Quantum and disorder effects in Davydov soliton theory. Physical Review A 1991; 44(4):2694-2708. doi:10.1103/PhysRevA.44.2694.

[93] K. Itoh, T. Shimanouchi. Vibrational spectra of crystalline formamide. Journal of Molecular Spectroscopy 1972; 42(1):86-99. doi:10.1016/0022-2852(72)90146-4.

[94] N. A. Nevskaya, Y. N. Chirgadze. Infrared spectra and resonance interactions of amideI and II vibrations of $\alpha$-helix. Biopolymers 1976; 15(4):637-648. doi:10.1002/bip. 1976.360150404 .

[95] L. D. Landau, E. M. Lifshitz. Quantum Mechanics. Vol. 3 of Course of Theoretical Physics. Pergamon Press, Oxford, 1965.

[96] D. D. Georgiev, J. F. Glazebrook. The quantum physics of synaptic communication via the SNARE protein complex. Progress in Biophysics and Molecular Biology 2018; 135:16-29. doi:10.1016/j.pbiomolbio.2018.01.006

[97] P. Jordan. Die Physik und das Geheimnis des organischen Lebens. Friedrich Vieweg \& Sohn, Braunschweig, Germany, 1941. https://repository.aip.org/islandora/ object/nbla:8133.

[98] R. H. Beyler. Targeting the organism: The scientific and cultural context of Pascual Jordan's quantum biology, 1932-1947. Isis 1996; 87(2):248-273.

[99] M. Born, R. Oppenheimer. Zur Quantentheorie der Molekeln. Annalen der Physik 1927; 389(20):457-484. doi:10.1002/andp.19273892002.

[100] J. P. Lowe, K. Peterson. Quantum Chemistry. 3rd Edition. Academic Press, Amsterdam, 2005.

[101] S. J. Ganesan, S. Matysiak. Role of backbone dipole interactions in the formation of secondary and supersecondary structures of proteins. Journal of Chemical Theory and Computation 2014; 10(6):2569-2576. doi:10.1021/ct401087a.

[102] L. Cruzeiro. The VES hypothesis and protein misfolding. Discrete and Continuous Dynamical Systems - Series S 2011; 4(5):1033-1046. doi:10.3934/dcdss.2011.4.1033.

[103] B. Alberts, D. Bray, K. Hopkin, A. D. Johnson, J. Lewis, M. Raff, K. Roberts, P. Walter. Essential Cell Biology. 4th Edition. Garland Science, New York, 2013. 
[104] E. J. Milner-White. Protein three-dimensional structures at the origin of life. Interface Focus 2019; 9(6):20190057. doi:10.1098/rsfs.2019.0057.

[105] R. R. Poznanski, L. A. Cacha, A. Z. A. Latif, S. H. Salleh, J. Ali, P. Yupapin, J. A. Tuszynski, T. M. Ariff. Molecular orbitals of delocalized electron clouds in neuronal domains. Biosystems 2019; 183:103982. doi:10.1016/j.biosystems.2019.103982

[106] L. S. Brizhik. Influence of electromagnetic field on soliton-mediated charge transport in biological systems. Electromagnetic Biology and Medicine 2015; 34(2):123-132. doi: 10.3109/15368378.2015.1036071.

[107] D. D. Georgiev. Monte Carlo simulation of quantum Zeno effect in the brain. International Journal of Modern Physics B 2015; 29(7):1550039. doi:10.1142/ S0217979215500393.

[108] A. Pross, R. Pascal. The origin of life: what we know, what we can know and what we will never know. Open Biology 2013; 3(3):120190. doi:10.1098/rsob.120190.

[109] N. Kitadai, S. Maruyama. Origins of building blocks of life: A review. Geoscience Frontiers 2018; 9(4):1117-1153. doi:10.1016/j.gsf.2017.07.007.

[110] D. H. Lee, K. Severin, Y. Yokobayashi, M. R. Ghadiri. Emergence of symbiosis in peptide self-replication through a hypercyclic network. Nature 1997; 390(6660):591594. doi:10.1038/37569.

[111] H. Duim, S. Otto. Towards open-ended evolution in self-replicating molecular systems. Beilstein Journal of Organic Chemistry 2017; 13:1189-1203. doi:10.3762/bjoc.13. 118 .

[112] P. Andras, C. Andras. The origins of life - the 'protein interaction world' hypothesis: protein interactions were the first form of self-reproducing life and nucleic acids evolved later as memory molecules. Medical Hypotheses 2005; 64(4):678-688. doi:10.1016/j . mehy.2004.11.029. 